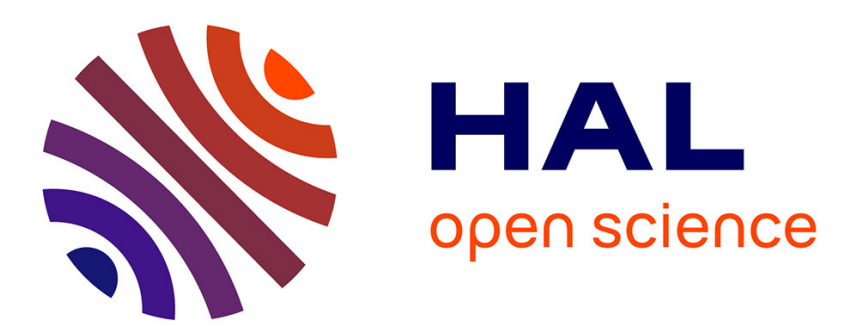

\title{
Modeling the Electrostatics of Hollow Shell Suspensions: Ion Distribution, Pair Interactions, and Many-Body Effects
}

\author{
Yannick Hallez, Martine Meireles
}

\section{- To cite this version:}

Yannick Hallez, Martine Meireles. Modeling the Electrostatics of Hollow Shell Suspensions: Ion Distribution, Pair Interactions, and Many-Body Effects. Langmuir, 2016, vol. 32 ( $\mathrm{n}^{\circ} 40$ ), pp. 1043010444. 10.1021/acs.langmuir.6b02730 . hal-01390506

\section{HAL Id: hal-01390506 https://hal.science/hal-01390506}

Submitted on 2 Nov 2016

HAL is a multi-disciplinary open access archive for the deposit and dissemination of scientific research documents, whether they are published or not. The documents may come from teaching and research institutions in France or abroad, or from public or private research centers.
L'archive ouverte pluridisciplinaire HAL, est destinée au dépôt et à la diffusion de documents scientifiques de niveau recherche, publiés ou non, émanant des établissements d'enseignement et de recherche français ou étrangers, des laboratoires publics ou privés. 


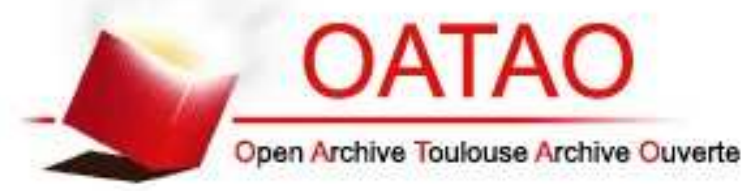

\section{Open Archive TOULOUSE Archive Ouverte (OATAO)}

OATAO is an open access repository that collects the work of Toulouse researchers and makes it freely available over the web where possible.

This is an author-deposited version published in : http://oatao.univ-toulouse.fr/ Eprints ID : 16211

To link to this article : DOI : 10.1021/acs.langmuir.6b02730 URL : http://dx.doi.org/10.1021/acs.langmuir.6b02730

To cite this version : Hallez, Yannick and Meireles, Martine Modeling the Electrostatics of Hollow Shell Suspensions: Ion Distribution, Pair Interactions, and Many-Body Effects. (2016) Langmuir, vol. 32 ( $\left.{ }^{\circ} 40\right)$. pp. 10430-10444. ISSN 0743-7463

Any correspondence concerning this service should be sent to the repository administrator: staff-oatao@ listes-diff.inp-toulouse.fr 


\title{
Modeling the Electrostatics of Hollow Shell Suspensions: Ion Distribution, Pair Interactions, and Many-Body Effects
}

\author{
Yannick Hallez* and Martine Meireles \\ Laboratoire de Génie Chimique, Université de Toulouse, CNRS, INPT, UPS, Toulouse 31000, France
}

\begin{abstract}
Electrostatic interactions play a key role in hollow shell suspensions as they determine their structure, stability, thermodynamics, and rheology and also the loading capacity of small charged species for nanoreservoir applications. In this work, fast, reliable modeling strategies aimed at predicting the electrostatics of hollow shells for one, two, and many colloids are proposed and validated. The electrostatic potential inside and outside a hollow shell with a finite thickness and a specific permittivity is determined analytically in the Debye-Hückel (DH) limit. An expression for the interaction potential between two such hollow shells is then derived and validated numerically. It follows a classical Yukawa form with an effective charge depending on the shell geometry, permittivity, and inner and outer surface charge densities. The predictions of the Ornstein-Zernike $(\mathrm{OZ})$ equation with this pair potential to determine equations of state are then evaluated by comparison to results obtained with a Brownian dynamics algorithm coupled to the resolution of the linearized Poisson-Boltzmann and Laplace equations (PB-BD simulations). The OZ equation based on the DLVO-like potential performs very well in the dilute regime as expected, but also quite well, and more surprisingly, in the concentrated regime in which full spheres exhibit significant many-body effects. These effects are shown to vanish for shells with small thickness and high permittivity. For highly charged hollow shells, we propose and validate a charge renormalization procedure. Finally, using PB-BD simulations, we show that the cell model predicts the ion distribution inside and outside hollow shells accurately in both electrostatically dilute and concentrated suspensions. We then determine the shell loading capacity as a function of salt concentration, volume fraction, and surface charge density for nanoreservoir applications such as drug delivery, sensing, or smart coatings.
\end{abstract}

\section{INTRODUCTION}

Colloidal hollow shells encapsulating smaller active or useful species are ubiquitous in the natural world. Some examples are cells, lipoproteins containing lipids, or viral capsids containing DNA. Thanks to the rapid advance of synthesis and assembly methods, it is now possible to create artificial hollow shells in a well-controlled manner. ${ }^{1,2}$ Several applications relying on these systems are expected to lead to strong societal or economic benefits in the domains of medicine, biotechnology, cosmetics, coating, sensing, microreaction, catalysis, crystallization, acoustics, electronics, optoelectronics, etc. ${ }^{1-3}$ Hollow shells are used for the encapsulation, vectorization, and/or controlled release of various substances in many of these domains, but they can also be considered for their own particular properties, for example for their low density or for their tunable plasmon resonance. $^{4}$

In this work, we consider porous, charged hollow shells because they can be used to encapsulate small charged species, they are naturally stabilized by their surface charges, and their structure in a suspension can be controlled through their mutual interactions, opening the way to many applications beyond encapsulation. Both the amount of matter trapped in capsules and the structure of the suspension can be tuned, for example by varying the surface charge density of the colloids, which can itself be controlled with the $\mathrm{pH}$ of the suspension. This type of hollow shell also provides some selectivity to the transfer of small species: capsules will retain almost only oppositely charged species and reject others (provided the suspension is not near close packing ${ }^{5}$ ). Examples of such porous, charged hollow shells are silica microcapsules, 6,7 metallodielectric shells, ${ }^{4}$ colloidosomes, ${ }^{8}$ and, to some extent, polyelectrolyte multilayer microcapsules, ${ }^{3}$ bilayer vesicles containing ion channels, or viral capsids. ${ }^{9}$ The small charged species able to migrate through these shells will be called ions in this article for simplicity, although ions are not the only type of charged species one would want to encapsulate.

Electrostatic interactions (EI) are central to this work at two fundamental levels which drive the microscopic and macroscopic behavior of the system. At the microscopic scale, electrostatic interactions between small ions and shells determine the amount of ions that can be "trapped" near the surface of the shell, and in particular inside this shell. The nanoreservoir aspect of a shell suspension is therefore intrinsically linked to ion-shell electrostatic interactions. On the other hand, the structure, stability, and the macroscopic behavior of the suspension, which is determined by its constitutive relations (equation of state, rheology), are strongly determined by shell-shell electrostatic interactions. These 
aspects are fundamental for predicting both equilibrium and nonequilibrium properties of shell suspensions. In equilibrium, controlling the structure can lead to interesting applications like photonic crystals. ${ }^{4}$ Out of equilibrium, shell-shell interactions influence the flow of suspensions in small capillaries or veins, or the transformation of suspensions during processes such as drying, filtration, or coating. These interactions are also essential for a determination of the nature and properties of composite materials fabricated by directed or self-assembly of hollow colloids such as viruses, with applications in domains such as electronics, sensing and biomedical engineering. ${ }^{10-12}$

The aim of this article is to provide useful guidelines for efficient, precise modeling of hollow shell suspensions. This modeling will enable the loading capacity of shells and their collective behavior to be predicted as a function of physical control parameters such as their charge, thickness, dielectric permittivity, or volume fraction, with a view to being able to design hollow shells with optimized properties for specific applications. Some of these applications require the assembly of colloidal crystals (e.g., to enhance tunable plasmon resonance ${ }^{4}$ ) or close packing to be achieved (e.g., to form self-healing coatings), which is a particular source of motivation to study the modeling of electrostatically concentrated suspensions, i.e. suspensions with interaction ranges comparable to, or larger than, the mean intercolloid distance. This is a domain unfavorable to the use of standard DLVO theories, which are built for dilute suspensions since they rely on isolated pair potentials, ignoring many-body effects.

In a mean-field picture, ion-shell and shell-shell interactions are both strongly determined by the electrostatic potential distribution in the N-body suspension. It is possible to compute the details of this field numerically, at great expense, for a few hundreds or a few thousands of colloids (computing all ion-shell and shell-shell interactions is also possible at the primitive model level, with the same numerical cost). However, designing and optimizing new colloidal systems based on their modeling requires the computation of the properties of many different suspensions with varying geometrical or physicochemical parameters, such as the size, charge and dielectric constant of the particles, or the salt content or volume fraction of the suspension. Due to the large number of degrees of freedom to be explored, using detailed simulations is not realistic. For this reason, colloidal engineering has to rely on approximate but fast models aimed at predicting the ion distribution, the structure, and the dynamics of colloidal suspensions. Several well-known and well tested models have been devised for full colloidal spheres. We will focus on two of them. The first one is the cell model $^{13}(\mathrm{CM})$ in which the $\mathrm{N}$-body suspension is split into $\mathrm{N}$ identical, spherical, electro-neutral Wigner-Seitz cells containing a single colloid. The N-body $3 \mathrm{D}$ problem is reduced to a 1body $1 \mathrm{D}$ problem, for which the full computation of the electrostatic potential can be performed at virtually no cost. The cell model provides two very interesting pieces of information. First, the ion distribution around one particle is determined, and second, the osmotic pressure of the suspension can be estimated when the dispersion is solid-like. This model does not, however, provide information about the structure of the suspension. In the second model considered, in integral equation (IE) theory, the structure of the suspension is predicted for a given pair potential of interaction. Thermodynamic quantities like the osmotic pressure can be deduced from this structure. In concentrated suspensions, the pair potential must be an effective potential (beyond the classical DLVO theory) accounting for many-body correlations.

Several authors have already considered the problem of modeling the electrostatics of hollow shells, sometimes with the approaches mentioned above. The picture of a single shell in an infinite medium has been applied, for example, to determine the influence of electrostatics on the size and properties of viral capsids made by the assembly of charged protein subunits. ${ }^{14,15}$ The cell model is also a single colloid model, but it accounts for the finite volume fraction of colloids in the suspension. This was important for example to study the ion distribution inside and outside a hollow shell ${ }^{5,16,17}$ or the formation of surfactant bilayer vesicles. ${ }^{18,19}$ At the two colloid level, the pair interaction potential between two infinitely thin shells with an arbitrary surface charge distribution has been derived analytically in the Debye-Hückel limit, ${ }^{20}$ and the mean force between two hollow shells with a finite thickness and the same permittivity as the electrolyte as been computed in the no-salt limit with Monte Carlo simulations. ${ }^{12}$ To the best of our knowledge, the pair interactions exerted between hollow shells with a finite thickness and a dielectric constant different from that of the electrolyte have never been computed, although, unlike in the full sphere case, these parameters influence ion-colloid and colloid-colloid interactions at first order. Neither are we aware of simulations including more than two hollow shells, either in a primitive model or in a mean-field context. In this article, we therefore extend previous works by considering porous and charged hollow shells with a finite thickness and with a dielectric permittivity different from that of the electrolyte.

In the first section, analytical results are derived in the Debye-Hückel limit concerning the electrostatic field generated by a single hollow shell in a Wigner-Seitz cell and concerning the pair interaction between two hollow shells in an otherwise infinite and empty medium. These results are validated numerically. In the second part, we evaluate suspension modeling strategies relying on these pair interactions (based on the Ornstein-Zernike equation here) and on the cell model to compute equations of state by comparison with data generated by more refined simulations. These simulations combine a classical Brownian dynamics algorithm with the resolution of the nonlinear PoissonBoltzmann and Laplace equations in an N-body system at each time step. Image effects due to dielectric discontinuities and many-body effects are thus fully included. In this second section, we will focus on two aspects in particular: the onset of many-body effects in concentrated suspensions, and the proposition of a charge renormalization method to compute the thermodynamics of highly charged hollow shell suspensions based on effective pair interactions. In the last part of this article, the modeling of the distribution of ions inside and outside hollow shells is discussed, and general conclusions are drawn concerning the use of such colloids as nanocontainers.

\section{DEBYE-HÜCKEL THEORY FOR ONE AND TWO SHELLS}

We consider the coarse-grained version of hollow shells depicted in Figure 1 and already used by several authors. ${ }^{5,14,21}$ The outer and inner surfaces of these shells are spheres of radii $a$ and $a^{\prime}$ and surface charge densities $\sigma$ and $\sigma^{\prime}$, respectively. The details of the structure of the shell wall are smoothed out, leading to a uniform material with effective dielectric permittivity $\epsilon_{s}$ usually lower than that of water. This model is probably not well suited to objects with thick, highly porous 

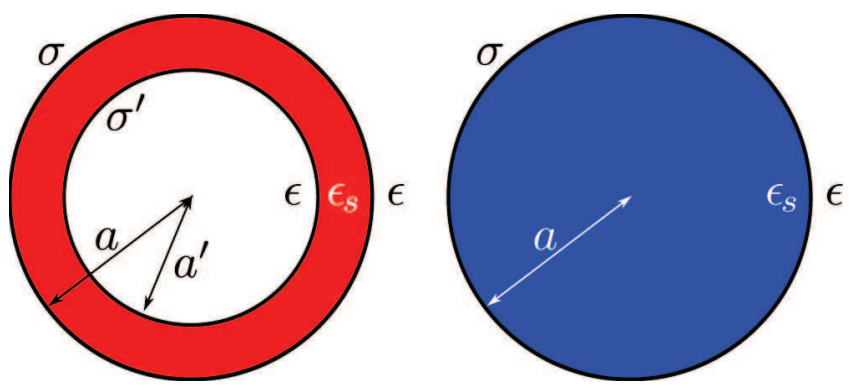

Figure 1. Coarse-grained versions of a hollow shell (left) and sphere (right) considered in this work.

walls like microgels since ions and charges may then be dispersed into the "solid" phase. A treatment of this problem can be found in refs 22 and 23, for instance. The present coarse-graining strategy is more suited to particles with impermeable walls punctured by a few passageways authorizing ion exchange, like colloidosomes, ${ }^{8}$ silica microcapsules, ${ }^{7,24}$ viral capsids, ${ }^{9}$ bilayer vesicles containing nonselective ion channels, or incomplete metallodielectric shells. ${ }^{4}$ The electrolyte is assumed to have a uniform dielectric constant $\epsilon$. The hollow shell suspension is taken to be in Donnan equilibrium with a salt reservoir with ion density $n_{0}$. Since the hollow shells are porous to microions, the electrolyte inside them is also in Donnan equilibrium with the salt reservoir.

Motivated by the large spatial and temporal scale separation between the microions and the colloids, we treat the hollow spheres explicitly and the microions at the mean-field level. Their influence on electrostatics is accounted for only through their local density, assumed to be given by the Boltzmann approximation

$$
n^{ \pm}=n_{0} \mathrm{e}^{\mp z^{ \pm} \psi}
$$

where $z^{ \pm}$is the valency of microions, $\psi=\Psi e / k T$ is the reduced electrostatic potential, $\Psi$ is the electrostatic potential, $e$ is the elementary charge and $k T \equiv 1 / \beta$ is the thermal energy. With this approach, we discard both the finite size of the ions and the correlations between them. This picture leads to an accurate description of the original system under the condition of low electrostatic coupling $\Xi=2 \pi \sigma z^{3} l_{\mathrm{B}}{ }^{2} / e \lesssim 1$, where $\sigma$ is the surface charge density, and $l_{\mathrm{B}}=e^{2} / 4 \pi \epsilon k T$ is the Bjerrum length. This condition is generally not respected when multivalent counterions are involved, but it is met in aqueous 1:1 electrolytes even for highly charged colloids. ${ }^{25}$ In this context, the Poisson equation for the electrostatic potential inside the fluid phase, both inside and outside the capsule, becomes the PoissonBoltzmann (PB) equation

$$
\nabla^{2} \psi=\sinh \psi
$$

where $\nabla$ is the gradient operator scaled by the inverse Debye length $\kappa=\left(2 n_{0} e^{2} / \epsilon k T\right)^{1 / 2}$. This equation being nonlinear, its resolution is impossible analytically and is extremely CPUexpensive numerically for a system including several colloids. Useful analytical results can however be obtained in the low electrostatic potential regime. In this case, the $\mathrm{PB}$ equation becomes the Debye-Hückel (DH) equation

$$
\nabla^{2} \psi=\psi
$$

Ions are taken to be mostly excluded from the shell wall, so the electrostatic potential there is the solution of the Laplace equation

$$
\nabla^{2} \psi=0
$$

Equations 2 and 4 are coupled on the inner and outer surfaces of the colloids by boundary conditions (BC). In this work, we consider the constant charge density $\mathrm{BC}$

$$
\left[\left.\mu \nabla \psi\right|_{\mathrm{s}}-\left.\nabla \psi\right|_{\mathrm{e}}\right] \cdot \mathbf{n}=\bar{\sigma}
$$

where $\mu=\epsilon_{\mathrm{s}} / \epsilon$ is the shell-to-electrolyte permittivity ratio, $\mathbf{n}$ is the unit vector normal to the colloid surface and pointing toward the electrolyte, and $\bar{\sigma}$ is the surface charge density scaled by $\sqrt{2 n_{0} \epsilon k T}$. Other boundary conditions might be used. Metallic surfaces are more amenable to modeling by a constant potential condition, and a number of materials actually exhibit charge regulation. ${ }^{26}$ In the latter case, the surface charge density depends on the $\mathrm{pH}$ of the suspension and one the salt concentration near the surface. Different charge regulation mechanisms exist, depending on the material of the colloids, on the solvent, and on the type of ions considered. Therefore, in order to keep the discussion as generic as possible, we chose to use a constant charge $\mathrm{BC}$ in this work.

The electrostatics problem (2)-(4)-(5) is driven by six dimensionless parameters in the $\mathrm{PB}$ theory. They are the volume fraction $\phi$, the reduced radius $\kappa a, \bar{\sigma}, \mu=\epsilon_{s} / \epsilon$, the coreto-shell radius ratio $\alpha=a^{\prime} / a$, and the inner-to-outer surface charge density ratio $\sigma^{\prime} / \sigma$. The influence of these parameters will be explored around a reference set of parameters provided in Table 1 . If the value of a parameter is not explicitly specified in this article, the value indicated in Table 1 has been used.

\section{Table 1. Default Parameters Used in This Work ${ }^{a}$}

\begin{tabular}{cll} 
symbol & \multicolumn{1}{c}{ quantity } & \multicolumn{1}{c}{ value } \\
$a$ & external radius & $14 \mathrm{~nm}$ \\
$a^{\prime}$ & internal radius & $11.2 \mathrm{~nm}$ \\
$\epsilon$ & solvent permittivity & $78 \epsilon_{0}$ \\
$\epsilon_{s}$ & solid permittivity & $2 \epsilon_{0}$ \\
$\alpha$ & inner-to-outer radius ratio & 0.8 \\
$\bar{\sigma}$ & external surface charge density & 0.25 \\
$\bar{\sigma}^{\prime}$ & internal surface charge density & 0.25 \\
$\mathrm{Zl}_{B} / a$ & external surface charge & 0.5 \\
${ }^{a} \epsilon_{0}=8.854 \times 10^{-12} \mathrm{Fm}^{-1}$ is the vacuum permittivity. & \\
\hline
\end{tabular}

In section 2.1 , we solve the system (3)-(4)-(5) analytically in a Wigner-Seitz cell. The benefit of this is two-fold, as we obtain both an expression for the osmotic pressure valid in electrostatically concentrated suspensions ${ }^{27}$ and the general spherically symmetric solution for the electrostatic potential and ion distribution inside and outside one shell. In section 2.2, we use the infinite dilution limit of this solution to derive an analytical expression for the interaction potential between two shells.

\subsection{Solution in the Spherical Wigner-Seitz Cell Model.} One hollow sphere is placed at the center of a spherical Wigner-Seitz cell of radius $R=a \phi^{-1 / 3}$, where $\phi=4 \pi a^{3} / 3 V$ is the volume fraction and $V$ is the total volume of the suspension. Note that $\phi$ is an apparent volume fraction, not the solid volume fraction $\phi_{s}=4 \pi\left(a^{3}-a^{\prime 3}\right) / 3 V$. In the DH theory, the electrostatic potential $\psi(\kappa r)$ is the solution of the Laplace (4) and $\mathrm{DH}$ (3) equations with the additional boundary conditions 


$$
\begin{array}{ll}
\psi^{\prime}(0) & =0 \\
\psi^{\prime}(\kappa R) & =0 \\
\psi\left(\kappa a^{\prime-}\right) & =\psi\left(\kappa a^{++}\right) \\
\psi\left(\kappa a^{-}\right) & =\psi\left(\kappa a^{+}\right) \\
-\mu \psi^{\prime}\left(\kappa a^{++}\right)+\psi^{\prime}\left(\kappa a^{\prime-}\right) & =\bar{\sigma}^{\prime} \\
\mu \psi^{\prime}\left(\kappa a^{-}\right)-\psi^{\prime}\left(\kappa a^{+}\right) & =\bar{\sigma}
\end{array}
$$

The first is a symmetry requirement. The second stems from Gauss's law and expresses the global electroneutrality of the cell. The third and fourth ensure the continuity of the potential across the inner and outer surfaces of the vesicle. The plus or minus superscripts indicate a value infinitesimally larger or smaller, respectively, than the superscripted quantity. The last two relations are the constant charge conditions (5) at these surfaces. The solution of this system is

$$
\psi(\kappa r)=\left\{\begin{array}{c}
A_{\text {in }} \frac{\sinh \kappa r}{\kappa r} \text { for } r<a^{\prime} \\
\frac{A_{s}}{\kappa r}+B_{s} \text { for } a^{\prime} \leq r<a \\
A_{\text {out }} \frac{j^{+}(\kappa r)}{\kappa r} \text { for } r \geq a
\end{array}\right.
$$

where $j^{ \pm}(x)=e^{x} \pm \delta e^{-x}, \delta=\frac{\kappa R-1}{\kappa R+1} \mathrm{e}^{2 \kappa R}$, and $A_{\text {in }}, A_{s}, B_{s}$, and $A_{\text {out }}$ are constants given by

$$
\begin{aligned}
& A_{\text {out }}=\frac{\mu\left[\bar{\sigma}(\kappa a)^{2}+\bar{\sigma}^{\prime}\left(\kappa a^{\prime}\right)^{2}\right]+g \bar{\sigma}(\kappa a)^{2}(1-\alpha)}{g \alpha\left(\mu j^{+}(\kappa a)+h\right)-h(\mu+g)} \\
& A_{\text {in }}=\frac{\bar{\sigma}^{\prime}\left(\kappa a^{\prime}\right)^{2}+\bar{\sigma}(\kappa a)^{2}+h A_{\text {out }}}{\kappa a^{\prime} \cosh \left(\kappa a^{\prime}\right)-\sinh \left(\kappa a^{\prime}\right)} \\
& \mu A_{s}=-h A_{\text {out }}-\bar{\sigma}(\kappa a)^{2} \\
& \kappa a^{\prime} \mu B_{s}=\mu \sinh \left(\kappa a^{\prime}\right) A_{\text {in }}+h A_{\text {out }}+\bar{\sigma}(\kappa a)^{2}
\end{aligned}
$$

where $g=\kappa a^{\prime} \operatorname{coth}\left(\kappa a^{\prime}\right)-1$ and $h=\kappa a j^{-}(\kappa a)-j^{+}(\kappa a)$. This solution is a generalization to finite volume fractions of the solution found by Šiber and Podgornik for an isolated capsule. $^{14}$

Compared to the solution for a simple sphere, the particularity of this solution is its dependence on the solid-tofluid permittivity ratio $\mu$ and on the core-to-shell radii ratio $\alpha$. The essential features of the electrostatic potential generated by hollow spheres are a high potential value in the core, and an enhanced field outside the particle (see Figure 2). These qualitative characteristics do not depend on the coarse-graining approximations, and have been observed repeatedly with different approaches. ${ }^{5,16}$ The high potential value inside the capsule is essentially due to the mutual interaction of the charges carried by the inner surface of the shell.

The effect of the permittivity ratio on the electrostatic potential is illustrated in Figure 2a for $\alpha=0.8$. For vanishingly small values of the permittivity of the wall, the potential field outside the particles reduces to the one generated by a full sphere with the same outer surface charge density. In this case, the solid phase completely screens the inner field from the outer field, and vice versa. If the wall permittivity is increased,

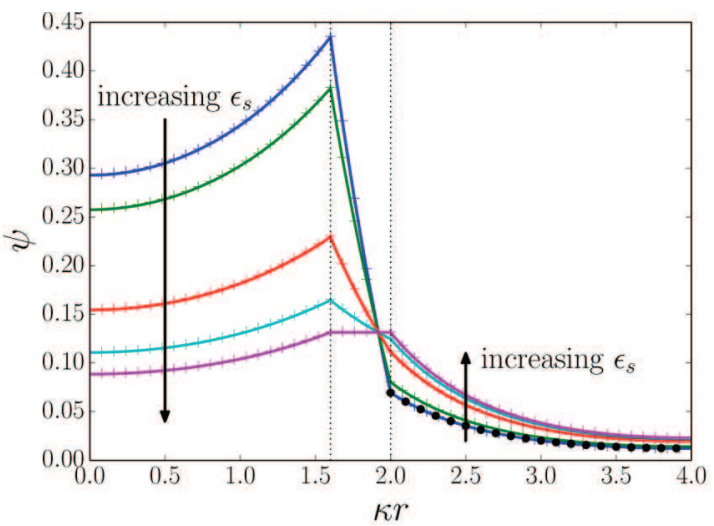

(a) $\epsilon_{s} / \epsilon_{0}=10^{-6}, 2,20,78$, and $10^{6}$ in the order indicated by the arrows, and $\alpha=0.8$.

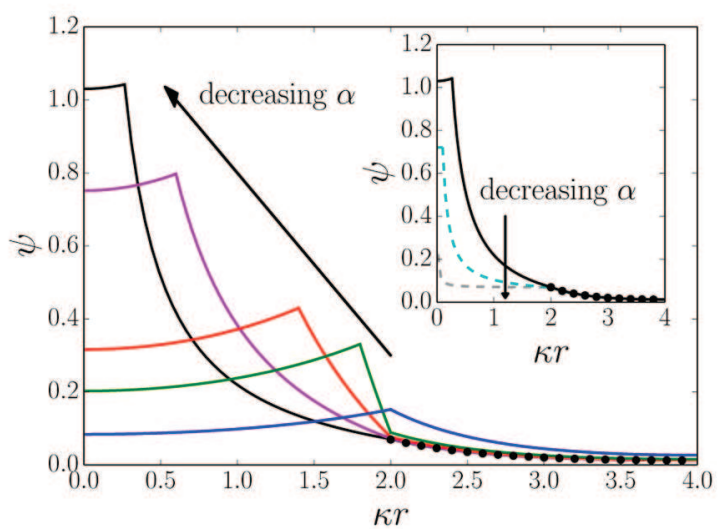

(b) In the main figure $\alpha=\alpha_{\max }, 0.3,0.7,0.9$, and $1-$ $10^{-6}$. In the insert $\alpha=0.01,0.05$, and $\alpha_{\max }$. Here $\alpha_{\max } \simeq 0.1327 . \epsilon_{s}=2 \epsilon_{0}$.

Figure 2. Electrostatic potential inside and outside a shell as a function of the solid relative permittivity $\epsilon_{s} / \epsilon_{0}$ and of the core-to-shell radii ratio $\alpha$. Lines: analytical solution (7)-(8); Crosses: numerical solution of (4)-(3)-(6). Black dots: full sphere solution, with the same outer surface properties. On both figures, $\kappa a=2, \epsilon=78 \epsilon_{0}, \bar{\sigma}=0.1, \bar{\sigma}^{\prime}=0.2$.

the inner potential decreases due to a reduced interaction between the inner charges. It nevertheless remains higher than the outer potential and contributes to its enhancement compared to the full sphere solution. Note that the outer solution presented in Figure 2a for a low shell permittivity $\epsilon_{s}=$ $2 \epsilon_{0}$ typical of many dielectrics immersed in water (green curve in Figure 2a) is very similar to the full sphere one. This is due to the moderate Debye length. For $\kappa a=0.5$, the external solution for the same shell permittivity is already close to the one obtained for a fully permeable wall (not shown).

The effect of the core-to-shell radii ratio $\alpha$ is exemplified in Figure $2 \mathrm{~b}$ for $\epsilon_{s} / \epsilon=2 / 78$. For large values of $\alpha$ (large core radius, main plot in Figure $2 \mathrm{~b}$ ), the potential increases as $\alpha$ decreases. The mechanism is similar to the one involved in the increase of the potential between two charged plates during their approach. However, for small core radii, the potential inside the core decreases whereas it would diverge between plates (see insert in Figure $2 \mathrm{~b}$ ). This is due to the reduction of the total charge carried by the inner surface with $a^{\prime}$. However, this particular behavior is of limited interest since the core radius is so small that the particles can hardly be considered as shells. As mentioned above, the high inner potential is 
responsible for the enhanced outer potential. The results presented in Figure $2 \mathrm{~b}$ show that this increase is more significant if the shell wall is thin, as expected.

To summarize, the inner potential field is increased if the shell permittivity is diminished or if the shell thickness is increased, which might prove useful for storing a maximum amount of counterions inside a capsule. Of course, the increase of the shell thickness would have to be performed for a constant inner radius in order to keep the volume available for ions constant. On the other hand, the external potential is enhanced for high shell permittivities and low shell thicknesses, which can be used to advantage if the aim is to build particles with very strong repulsions. Very thin shells with a surface charge density imposed by the physicochemical conditions in the electrolyte can experience a doubling of their effective charge compared to full spheres, and so a quadrupling of their interaction potential as will be shown later. This may be interesting for the design of strongly stabilized suspensions or colloidal glasses or crystals.

2.2. Pair Interactions. The external field around an isolated capsule can be obtained either from the $\phi \rightarrow 0$ limit of (7)-(8), or by following a procedure similar to the one employed in section 2.1, with the second boundary condition in (6) being replaced by the constraint of keeping the solution finite at all $r$. The solution is

$$
\psi_{0}(\kappa r)=\bar{\sigma}^{*}(\kappa a)^{2} \frac{\mathrm{e}^{\kappa a}}{1+\kappa a} \frac{\mathrm{e}^{-\kappa r}}{\kappa r}
$$

where

$$
\bar{\sigma}^{*}=\bar{\sigma}\left\{1+\mu \frac{\frac{\sigma^{\prime}}{\sigma} \alpha^{2}(1+\kappa a)-\alpha g}{\mu(\alpha g+1+\kappa a)+g(1-\alpha)(1+\kappa a)}\right\}
$$

Interestingly, the potential around an isolated shell takes the form of the potential around a full sphere (9) with the same outer radius $a$, the same screening length $\kappa^{-1}$, and an effective surface charge density $\sigma^{-*}$ given by relation (10). The ratio in relation (10) is a measure of the departure from the classical full sphere solution. Note that if the inner radius of the shell $a^{\prime}$ tends to zero, this ratio decreases smoothly and vanishes. Hence, the full sphere solution is obtained as the limit of the shell solution for a vanishingly small core radius. This property is not completely intuitive and is not verified in the WignerSeitz cell for instance. If the shell wall becomes impermeable to the electric field, this ratio also vanishes, leading once again to the classical full sphere solution as expected.

Pair interactions between colloidal particles in an infinite and empty medium can be estimated from the electrostatic potential (9) by invoking the linear superposition approximation (LSA). ${ }^{28,29}$ This procedure leads to the well-known screened-Coulombic potential of interaction

$$
\beta \omega=Z^{* 2} l_{\mathrm{B}}\left[\frac{\mathrm{e}^{\kappa a}}{1+\kappa a}\right]^{2} \frac{\mathrm{e}^{-\kappa r}}{r}
$$

where $Z^{*}=\sigma^{*} 4 \pi a^{2} / e$ and $\sigma^{*}$ is given by relation (10). It is important to note that the permittivity jump $\mu$ and the shell finite thickness accounted for by $\alpha \neq 1$ in (10) have an effect at this zeroth order for hollow shells, whereas $\mu$ appears only in image corrections for full spheres. Obtaining such a pair potential for hollow shells is of great practical interest since it permits the use of analytical and numerical methods routinely employed for suspensions of spheres at the minimal expense of replacing the sphere's surface charge by the effective charge $Z^{*}$.

In order to validate the pair potential $(10)-(11)$, we performed full 3D numerical computations of the electrostatic potential generated by a pair of particles in the $\mathrm{DH}$ framework (3) $-(4)-(5)$. Pictures of the electrostatic potential field are provided in Figure 3. The interaction potential was measured as

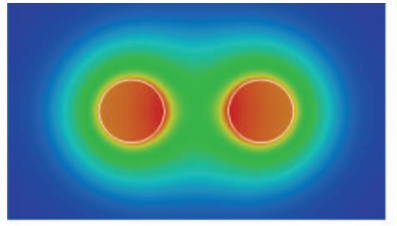

(a) $\kappa a=0.5, \psi \in[0,0.4]$.

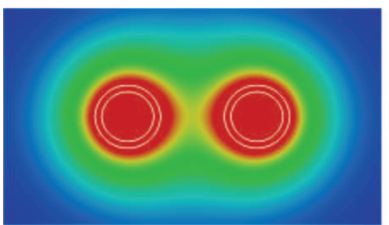

(c) $\kappa a=0.5, \psi \in[0,2.5]$.

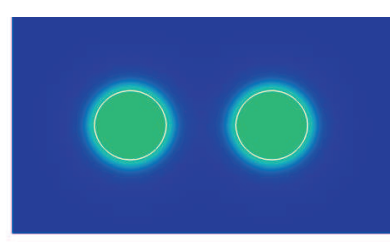

(b) $\kappa a=2, \psi \in[0,0.2]$.

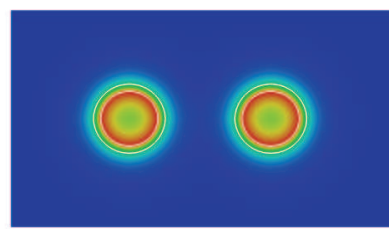

(d) $\kappa a=2, \psi \in[0,0.5]$.
Figure 3. Electrostatic potential map in a system of two spheres (top) or two shells (bottom). The colormap is linear with minimum value in blue and maximum value in red. $\epsilon_{\mathrm{s}} / \epsilon=2 / 78, \bar{\sigma}=\bar{\sigma}^{\prime}=1$.

the free energy variation observed when colloids were brought from infinity to a separation distance $r$

$$
\beta \omega=\sum_{i=1,2} \int_{S_{i}} \frac{1}{2} \frac{\sigma}{e} \psi \mathrm{d} S-2 \int_{S_{1}} \frac{1}{2} \frac{\sigma}{e} \psi_{0} \mathrm{~d} S
$$

where the indexes 1,2 identify the two colloids and the surfaces $S_{i}$ of each colloid include both the inner and outer surfaces for shells. Note that the interaction potential obtained in these simulations includes all image effects naturally, while the LSA solution (11) does not.

In Figure 4, the comparison between the analytical relation (11) (dashed lines) and the numerical data obtained from (12) (symbols) is presented for $\kappa a=0.5$ and $2, \mu=2 / 78$ and 1 , and for $\bar{\sigma}=\bar{\sigma}^{\prime}=1$ (we will assume $\bar{\sigma}=\bar{\sigma}^{\prime}$ in the rest of this article for simplicity). The small discrepancy observed between these results in Figure $4 b$ is due to image effects, and adding the first term of the correction for image interactions $\beta \omega_{1}$ to (11) following Ohshima's work $^{30}$ reconciles the theoretical pair potentials with the numerical results for spheres almost perfectly (dark blue continuous lines in Figure 4). Note that even if a shell is virtually identical to a sphere with charge $Z^{*}$ within the LSA, this is not true for image interactions since the latter depend on the boundary conditions on the inner and outer shell surfaces. The first image interaction between shells is thus by no means strictly given by the correction $\beta \omega_{1}$ for spheres. However, the sum $\beta \omega+\beta \omega_{1}$ computed with $Z^{*}$ is represented anyway as continuous light red lines in Figure 4 as a qualitative, ad-hoc, estimate of the corrected potential. This estimate actually seems rather accurate considering the excellent agreement between $\beta \omega+\beta \omega_{1}$ and the numerical data for shells in Figure 4.

The inserts in Figure $4 a$,b show the pair potential scaled by $Z^{2}$, where $Z$ is either the charge of a sphere or $Z^{*}$ for a shell. Without image effects, all curves collapse on the same master curve (dashed line). Any divergence from this master curve is 


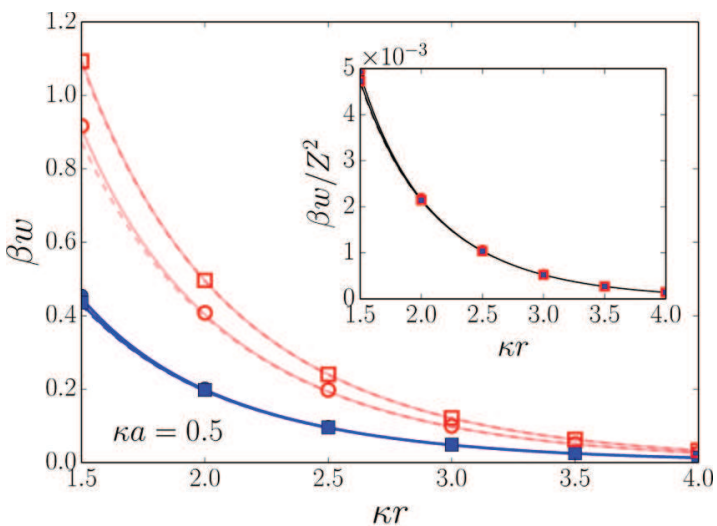

(a) $\kappa a=0.5$

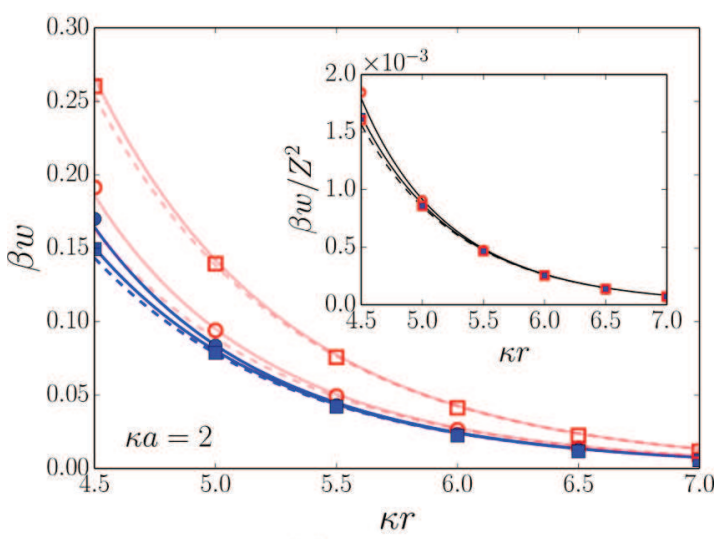

(b) $\kappa a=2$

Figure 4. Pair potential for spheres (dark blue) and shells (light red) for $\kappa a=0.5$ and $\kappa a=2$. Dashed lines: Yukawa potential (11); Solid lines: Yukawa potential (11) corrected with the first term in the infinite series expansion for the image effects for spheres; ${ }^{30}$ Symbols: 3D PB simulations with $\mu=2 / 78$ (circles) and $\mu=1$ (squares). The surface charge density for spheres is the same as the outer surface charge density of shells.

only due to image effects and is a function of the permittivity ratio $\mu$. The inserts in Figure 4 show that image interactions are negligible for long-range interactions $(\kappa a=0.5$, Figure $4 a)$ and slightly visible only at low solid permittivity for short-range interactions and short separation distances $(\kappa a=2$, Figure $4 \mathrm{~b})$. Therefore, we can conclude that image effects are not more important for shells than for full spheres, and that they can be ignored in practice.

Interesting qualitative features of the pair potential between shells can be pointed out from the data represented in Figure 4. First, the interaction potential between shells is always higher than that between spheres with the same outer surface charge density. This is to be expected since the inner charges of shells also contribute to the outer electrostatic potential and to the interaction potential. This effect is enhanced if the shell wall permittivity is increased. It is important to note that ignoring the inner charges would be reasonable only if the two conditions of short-range interactions and low wall permittivity were met simultaneously (see Figure $4 \mathrm{~b}$ ). Similarly, assuming a shell to be equivalent to a sphere with total charge $Z_{T}=4 \pi a^{2} \sigma /$ $e+4 \pi a^{\prime 2} \sigma^{\prime} / e$ is, in general, false. It would lead to reasonable agreement only in the long-range/high wall permittivity case displayed in Figure 4a. Relation (10), providing the correct expression of the effective charge as a function of $\alpha$ and the permittivity jump $\mu$, is therefore of prime importance for modeling general suspensions of capsules.

To conclude this part, we have shown that, within the linear superposition approximation, the pair interaction potential between shells follows a classical Yukawa form (11) with an effective charge $Z^{*}=4 \pi a^{2} \sigma^{*} / e$, where the dimensionless surface charge density $\sigma^{-*}$ is given by (10). Full 3D Laplace/Linearized Poisson-Boltzamnn simulations confirm this form, and show additionally that image charge effects are not more important than for full spheres, and thus quite negligible in practice. The pair potential (11) can therefore be used in classical liquid theories and in standard simulation software (MD, MC, BD) to compute the structure of a suspension, its stability, rheology, and equation of state within the one component model. The robustness of this approach for computing equations of state is investigated in the next section, with emphasis on many-body effects in concentrated hollow shell suspensions.

\section{SUSPENSION THERMODYNAMICS}

The macroscopic properties of a suspension are largely influenced by colloid-colloid interactions. In the previous section, we derived pair interactions for hollow shells in the $\mathrm{DH}$ limit. The aim of the present section is partly to evaluate the use and performances of these DLVO-like potentials when plugged into the integral equation (IE) theory to predict the equation of state (EOS) of weakly charged hollow shell suspensions, and partly to perform the same evaluation for effective pair potentials derived with a renormalization procedure in the high charge regime. The quality of the predictions of the cell model will also be discussed. The osmotic pressures obtained with these models are compared with more accurate, but computationally demanding, results obtained with a homemade Brownian dynamics code coupled to the resolution of the many-body Laplace/PB eqs (2)-(4)(5) or Laplace/DH eqs (3)-(4)-(5) throughout a triperiodic computation box at each time step (PB-BD approach). ${ }^{27}$ In brief, the advantages of this method are that many-body image interactions and all periodic images of the simulation domain are naturally and exactly included due to the periodicity of the computed electrostatic potential field (in particular, no cutoff distance and no Ewald summation techniques are involved), the ion thermodynamics correspond directly to the grandcanonical ensemble due to the writing of the PB equation, and the simulation cost is not too dependent on the number of ions in the system since they are represented at a mean-field level, so colloidal dispersions with a significant amount of salt can be simulated.

The EOS is chosen to evaluate the different modeling strategies rather than other quantities, like a diffusion coefficient or rheological properties, because it is an equilibrium property and since there are well established methods for computing it for full spheres. The radial distribution function is often chosen as a reference result to compare models, but we do not make this choice here. The reason is two-fold: first, the cell model, useful and precise in solid-like suspensions, does not predict a structure and, second, gathering statistics on the structure that are clean enough to permit a discriminating comparison with integral equation theory is difficult with the limited number of hollow shells that can be included in PB-BD simulations.

Computing the osmotic pressure in the cell model is straightforward, as it is given as an explicit function of the electrostatic potential at the cell boundary $\psi_{R}$. In the PB theory 


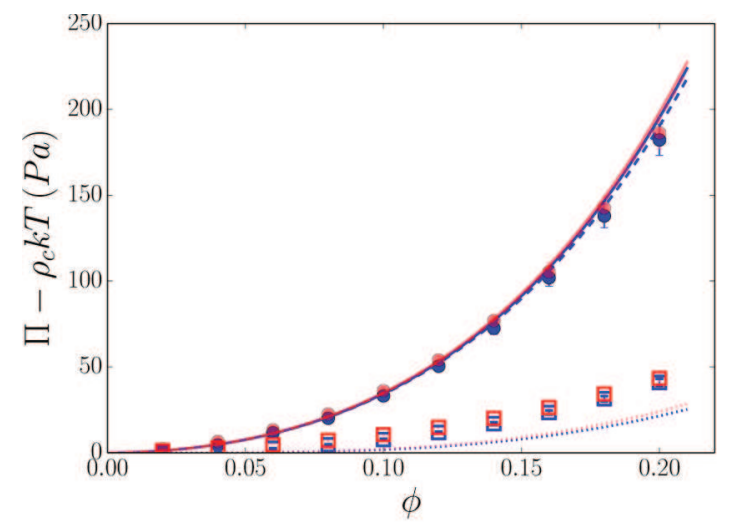

(a) $\kappa a=2, \epsilon_{s}=2$.

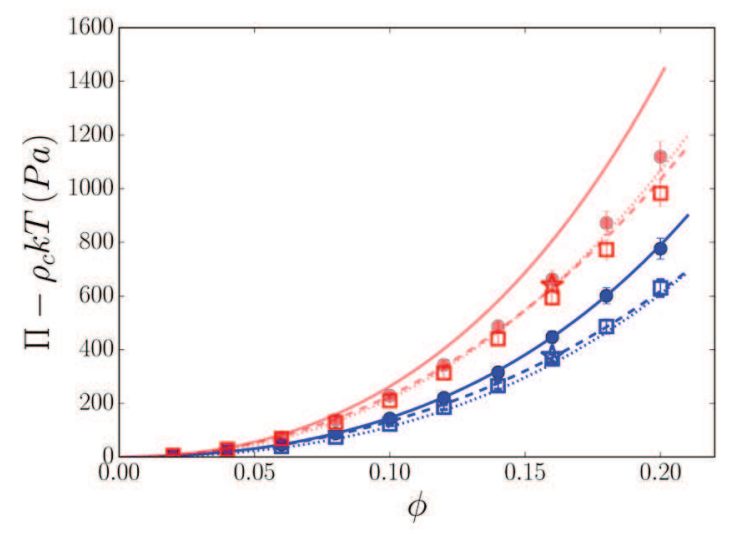

(c) $\kappa a=0.5, \epsilon_{s}=2$.

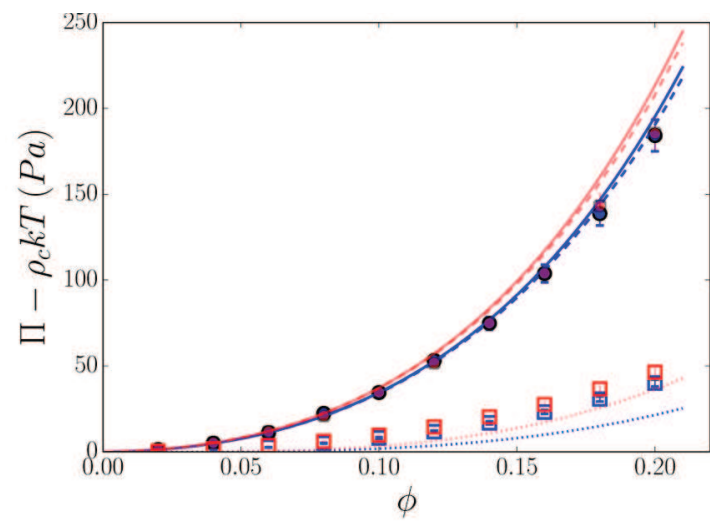

(b) $\kappa a=2, \epsilon_{s}=78$.

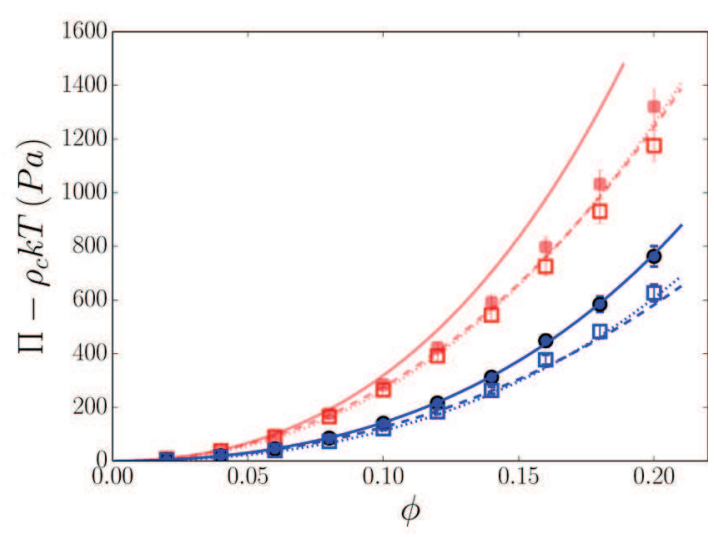

(d) $\kappa a=0.5, \epsilon_{s}=78$.

Figure 5. Nonideal part of the osmotic pressure computed from DH-BD simulations (circles), OZ-RY theory with standard Yukawa potential (11) (dashed lines), OZ-RY theory with corrected Yukawa potential (13) (solid lines), and the cell model (dotted lines) for full spheres and hollow shells. Squares: same as the circles but with the electrostatic contribution only (the contribution from hard core contacts is not included). Stars at $\phi=0.16$ in panel c: molecular dynamics simulations (LAMMPS) based on the standard Yukawa potential (11). Data related to full spheres appear in dark blue, and data related to hollow shells appear in light red.

it reads $\Pi=2 n_{0} k T\left(\cosh \psi_{R}-1\right)$, and in the $\mathrm{DH}$ theory it becomes $\Pi=2 n_{0} k T \psi_{\mathrm{R}}^{2} / 2$ when the PB equation is linearized around $\psi=0 .{ }^{31}$ We also consider pressure predictions obtained by solving the Ornstein-Zernike (OZ) equation with a Rogers-Young (RY) closure known to produce accurate results for hard-sphere-Yukawa potentials. With this method, either the "simple" pair potential (10)-(11) or a corrected pair potential, which will be described later, is used. Finally, the osmotic pressure in PB-BD simulations can be obtained thanks to an expression derived from the virial equation and containing surface and volume integrals of functions of the electrostatic potential field (see Appendix A for more details). A last remark is in order before presenting the results: for numerical reasons associated with the PB-BD algorithm, the radius used for hard core interactions is an effective radius $a+0.5 \delta$, which is at most $1.15 a$ in this work, while the radius for electrostatics is indeed $a$. The same shifted hard-core radius was used in the OZ-RY approach for consistency.

3.1. Equation of State in the Low Charge Regime. Equations of state (EOS) were computed in the DH framework with the three aforementioned methods for full spheres and hollow shells, with the parameters provided in Table 1. Two reservoir salt concentrations were investigated, corresponding to a low-salt regime with $\kappa a=0.5$ and to a high-salt regime with $\kappa a=2$. Two values of the solid phase permittivity were considered, a small one typical of many materials constituting colloidal particles, $\epsilon_{\mathrm{s}}=2$, and a large one equal to the dielectric constant of water $\epsilon_{\mathrm{s}}=78$.

The EOS of suspensions with short-range interactions are depicted in Figure 5a,b. Unsurprisingly, the cell model (dotted lines) significantly under-predicts the pressure. In fact, by construction, it attempts to predict the electrostatic contribution to the pressure only, without accounting for the collisional one. The electrostatic contribution is bound to be a minor one for the present weak, short-range electrostatic interactions, as confirmed by its measurement in the $\mathrm{DH}-\mathrm{BD}$ simulations reported in Figure 5a,b (squares). The pressure values predicted by the cell model are indeed of the same order of magnitude as their equivalent from DH-BD simulations, although not perfectly equal, as shown in previous works. $^{27,32-34}$ On the other hand, the integral equation theory based on the classical Yukawa potential (11) (dashed lines) perform very well for both spheres and hollow shells, provided the effective surface charge density (10) is used for the latter. This was expected in this electrostatically dilute, low-charge regime perfectly suitable for DH-like approaches.

The case of suspensions exhibiting long-range interactions is more thrilling. The EOS of suspensions of spheres and shells with $\kappa a=0.5$ are reported in Figure $5 c$,d. The cell model is able to capture the electrostatic contribution to the pressure quite satisfactorily, as expected in this electrostatically concentrated regime ${ }^{27,32-34}$ (compare the dotted lines and squares in Figure 
$5 c, d)$. It still lacks, however, the small contribution of collisional interactions necessary to obtain the full osmotic pressure. Better predictions can be obtained with the integral equation theory provided the correct pair potential is used. For suspensions of spheres (dark blue data), the IE theory with the standard Yukawa potential (dashed lines) is unable to predict correct values of the osmotic pressure in concentrated suspensions, here for $\phi>0.10$ (dark blue circles). This discrepancy is due to a many-body effect. It is related to the perturbation of the EDL of a given couple of spheres by other neighboring particles, in particular by the exclusion of ions from the solid volume of the latter. This effect is most present for long-ranged interactions and concentrated suspensions, when at least one additional colloid is "embedded" in the double layers of the pair of colloids. In this case, the effective pair potential for spheres depends on their local concentration (see ref 35 and 36 and references therein). It still follows the screened Coulombic form

$$
\beta \omega=Z^{2} l_{\mathrm{B}} X^{2} \frac{\mathrm{e}^{-\kappa r}}{r}
$$

but with a factor

$$
\begin{gathered}
X=\mathrm{e}^{\kappa a}\left[1+\kappa a+\mathrm{e}^{-\kappa a}(\sinh \kappa a-\kappa a \cosh \kappa a)\right. \\
\left.\rho_{\mathrm{c}} \int_{0}^{\infty} g(r) \frac{e^{-\kappa(r-2 a)}}{\kappa r} \mathrm{~d} \mathbf{r}\right]^{-1}
\end{gathered}
$$

depending on the colloidal density $\rho_{\mathrm{c}}$ and the colloidal radial distribution function $g(r)$. This effective pair potential reduces to the standard electrostatic contribution of the DLVO potential when $\rho_{\mathrm{c}} \rightarrow 0$. The prediction of the OZ-RY theory based on the corrected, effective, pair potential (13) (continuous lines in Figure 5) is in excellent agreement with DH-BD simulation data for spheres. This confirms that we actually observe the effect of many-body colloid-colloid correlations. Figures $5 \mathrm{c}$ and $5 \mathrm{~d}$ show this effect to be important for $\phi>0.10$, which corresponds to a scaled surface-to-surface distance $\kappa d=2 \kappa a\left(\phi^{-1 / 3}-1\right) \approx 1$, in line with the idea that many-body effects appear when EDL and colloid cores compete for the same volume.

DH-BD simulations were also conducted for a fixed volume fraction $\phi=0.18$ and various interaction ranges $\kappa a$ between 0.25 and 2. First, the results reported in Figure 6 confirm that

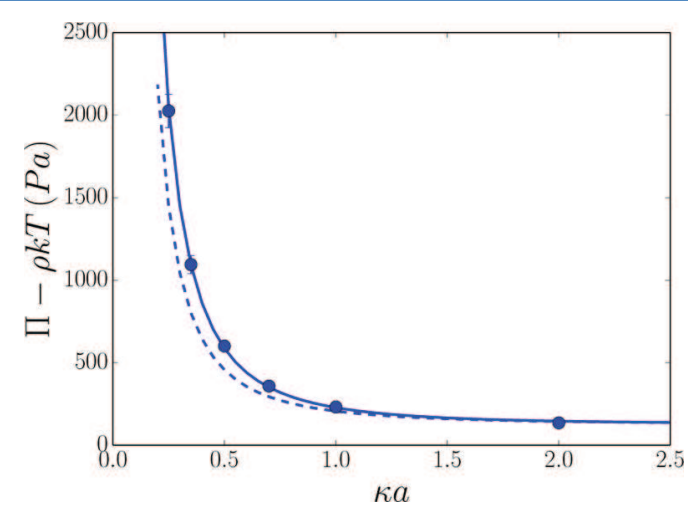

Figure 6. Nonideal part of the osmotic pressure computed from DHBD simulations (circles), OZ-RY theory with standard Yukawa potential (11) (dashed lines), OZ-RY theory with corrected Yukawa potential (13) (solid lines). Parameters $a, \epsilon, \epsilon_{\mathrm{s}}$, and $Z l_{\mathrm{B}} / a$ are those given in Table 1 , and $\phi=0.18$. very nice agreement can be obtained between the OZ-RY theory with the corrected potential (13) and the fully resolved DH-BD simulations. They also show the onset of many-body effects for interactions having a long range compared to the interparticle distance, here $\kappa a<0.8$, corresponding, once again, to $\kappa d \approx 1$.

This short account of possible ways to compute the EOS of concentrated suspensions of full spheres and the associated shortcomings summarizes ideas that can be found in the literature. It is, however, a useful reference for the following discussion about the modeling of concentrated hollow shell suspensions.

The EOS for suspensions of hollow shells with long-range interactions $\kappa a=0.5$ is displayed in Figure 5c,d in light red for $\alpha=0.8$. The cell model performs better here than for full spheres mainly because the repulsions are stronger for hollow shells, as shown in Figure 4. The collisions are thus less frequent for shells than for spheres and the electrostatic contribution captured by the cell model becomes the only relevant one.

For this system, unlike for full spheres, the agreement between $\mathrm{DH}-\mathrm{BD}$ simulations, the IE theory with the standard potential (11) and the cell model is very good! Quite strikingly, the many-body correction to the pair potential seems unnecessary for the present system of hollow spheres. As the walls of the shells are porous to microions, and, to some extent, to the electric field, ions can move inside other shells even in concentrated regimes. As a result, the EDL of a given pair of colloids is not as perturbed by the presence of a third particle as in the case of full spheres. This picture suggests that the performance of the OZ-RY theory with standard Yukawa potential (11) should improve when the thickness of shell walls is decreased $(\alpha \rightarrow 1)$ and when the permittivity of shells $\epsilon_{s}$ is increased.

This is confirmed in Figure 7, which shows the relative error $\mid \Pi_{\mathrm{DH}-\mathrm{BD}}-\Pi_{\mathrm{OZ}-\mathrm{RY}} \mathrm{l} /\left(\Pi_{\mathrm{DH}-\mathrm{BD}}-\rho k T\right)$ as a function of $\alpha$ for $\phi=$

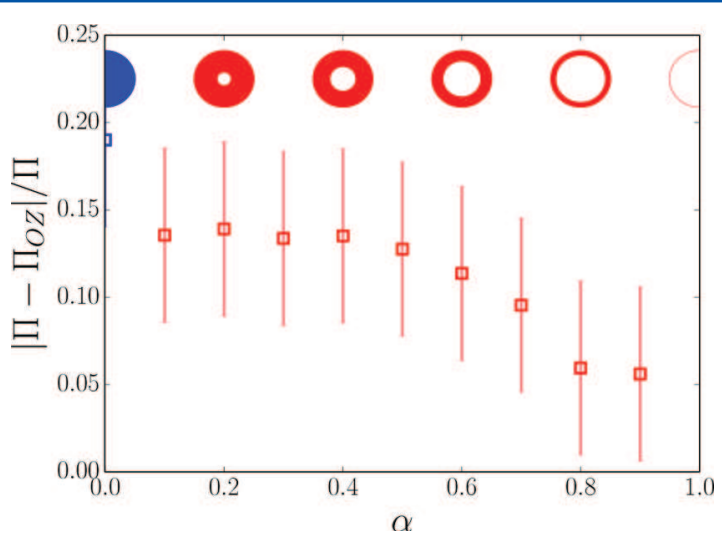

Figure 7. Relative error $\mid \Pi_{\mathrm{DH}-\mathrm{BD}}-\Pi_{\mathrm{OZ}-\mathrm{RY}} \mathrm{l} /\left(\Pi_{\mathrm{DH}-\mathrm{BD}}-\rho k T\right)$ on the osmotic pressure of a suspension of hollow spheres (light red symbols) and full spheres (dark blue symbols) at volume fraction $\phi=0.18$ and for $\kappa a=0.5$ and $\epsilon_{\mathrm{s}}=2 \epsilon_{0}$. The standard Yukawa potential (11) with the effective shell charge (10) is used. No many-body corrections are applied.

$18 \%, \kappa a=0.5$, and $\epsilon_{\mathrm{s}}=2 \epsilon_{0}$. This error is between 0 and $15 \%$ while the numerical error of the DH-PB method is of the order of $5 \%$ of the pressure value, making it delicate to draw very quantitative conclusions. We can see qualitatively however that the error decreases as the shell becomes thinner as anticipated. 
Note that the error saturates for small values of $\alpha$ instead of reaching that of a full sphere, indicating that the full sphere does not correspond to the asymptotic limit $\alpha \rightarrow 0$, at least in the DH-BD theory.

Three values of the shell dielectric constant were considered for $\phi=0.18, \alpha=0.8$, and $\kappa a=0.5$. Results for $\epsilon_{\mathrm{s}}=2 \epsilon_{0}$ and $78 \epsilon_{0}$ are reported in Figure $5 \mathrm{c}$,d. They show many-body corrections to the pressure of the order of $5.9 \%$ for $\epsilon_{s}=2 \epsilon_{0}$ (see Figure 7) and $4.6 \%$ for $\epsilon_{s}=78 \epsilon_{0}$. Another simulation was performed for the same system, but with an (unrealistic) vanishingly small dielectric constant $\epsilon_{\mathrm{s}}=10^{-6} \epsilon_{0}$. The pressure obtained is in very good agreement with a full sphere model including the manybody core exclusion correction (of the order of $20 \%$ ). This might seem surprising at first since the core of these shells is indeed accessible to small ions, and even contains a lot of counterions. The reason is that, for $\epsilon_{\mathrm{s}}=0$, the constant charge boundary conditions (5) no longer couple the internal and external fields, and the electrostatic potential outside shells is thus exactly the one that would be observed for full spheres with the same external surface charge (see Figure 2a). Moreover, the vanishing permittivity of shells prevents the field generated by a pair of particles 1 and 2 from penetrating into the core of a third particle, so their EDL are indeed excluded from the hollow core of this third particle. This is why the volume exclusion model designed for full spheres (13) works so well for hollow shells with vanishingly small permittivity. There are thus two effects contributing to manybody effects in hollow shell suspensions: the exclusion of ions from the particles is due partly to solid exclusion by the shell wall which depends on the inner-to-outer radii ratio $\alpha$, and partly to the screening of the external electric field by the shell material, which is determined by the dielectric constant of the shell $\epsilon_{s}$.

To conclude, we have shown that the equations of state of moderately charged suspensions of hollow shells can be modeled in manners quite similar to what is usually done for full spheres, with the need to avoid mostly the same shortcomings. In brief, the cell model should only be used with high surface charges and intercolloid distances shorter than the Debye length so that the structure of the suspension is solid-like, while the integral equation theory based on simple $\mathrm{DH}$-like - or DLVO - potentials should be used in exactly the opposite conditions. There are, however, a few distinctions between hollow shells and spheres, which we summarize here: (i) the cell model must obviously be adapted to the shell boundary conditions, (ii) the integral equation theory can be employed with a screened Coulombic potential provided the total charge is replaced by the effective charge given by relation (10), and (iii) many-body effects at high volume fraction are weaker for hollow shells than for full spheres, decrease when the shell thickness is decreased, and decrease when the shell permittivity is increased. Since these effects represent modifications of the osmotic pressure by only a few percent or a few tens percent for spheres, they might be simply and safely ignored for most hollow (thin-walled) shell systems of applicative interest.

3.2. Equation of State in the High-Charge Regime. The surface charge density considered in the previous section was intentionally taken to be low in order to ensure a straightforward comparison between the integral equation theory and the many-body simulations. In this case, the LSA employed to derive the pair potential (10)-(11) and the pair additivity hypothesis are justified, although image effects should be taken into account for a perfect comparison with a manybody DH-BD simulation.

However, the surface charge density of colloids with an applicative interest may be quite large, and linearizing the PB equation may not be justified in these systems. Electrostatic forces exerted on colloids are then nonadditive in general (see e.g. ref 37 for a measurement of three-body effects between spheres). If the suspension is not too electrostatically concentrated (i.e., the typical intercolloid distance is larger than the Debye length), it is still possible to use DebyeHückel-like theories like the one developed above, but with an effective or renormalized surface charge and an effective Debye length. The idea is that the long-distance tail of the potential field generated by one colloid has decreased enough for the potential to be lower than 1 , so this tail can be modeled with rescaled $\mathrm{DH}$ equation and boundary conditions. The effective interactions deriving from this $\mathrm{DH}$-like picture are then assumed to remain pairwise additive. Experimentally, the effective charge and Debye length are generally obtained by fitting structure factors computed from models, such as the integral equation theory, onto structure factors obtained by light or neutron scattering. It is also possible to compute effective parameters theoretically but there are many different approaches leading to different values. ${ }^{38}$ Although these renormalization procedures still do not give effective charges in perfect agreement with experimentally determined ones, they generally produce quite reasonable results. In this section, we show how it is possible to map the interactions between highly charged hollow shells onto a system of full spheres with effective parameters. We then validate this procedure against the results of many-body nonlinear PB Brownian dynamics simulations.

We follow the spirit of the method based on the cell model proposed by Alexander and co-workers ${ }^{39}$ and revisited by Trizac et al. ${ }^{40}$ In this approach, the effective parameters are chosen so that the potential on the outer cell boundary obtained analytically with the $\mathrm{DH}$ theory is matched to the potential computed numerically with the nonlinear PB theory. The only difference between the original cell model renormalization and the one used here is that the nonlinear simulation has to be conducted for a shell while the DH solution used is that of a sphere, as illustrated in Figure 8a. It would be possible to perform the same procedure with the $\mathrm{DH}$ solution for a shell (7) but the calculation of the effective charge would be more complicated and bring no additional insight, since the aim of such a procedure is to provide an effective pair potential to compute the structure and thermodynamics of a suspension, so that the details of the fields inside shells can be forgotten.

In order to validate this approach, the osmotic pressure was computed from a nonlinear PB-BD simulation for the parameters indicated in the legend of Figure 8. This is the reference pressure which should be obtained by renormalized $\mathrm{DH}$-like theories. The predictions of nonlinear PB-BD simulations, OZ-HNC theory with and without renormalization, and the PB and DH cell model are reported in Figure $8 \mathrm{~b}$. Here we used the HNC closure instead of the RY one because our implementation of the latter tended to be unstable. HNC is still expected to be quite precise for this strongly chargestabilized system. As expected, the use of DH-like theories without renormalization yields strongly overestimated osmotic pressures. The nonlinear cell model performs quite well for the present set of parameters. This was expected since the effective 


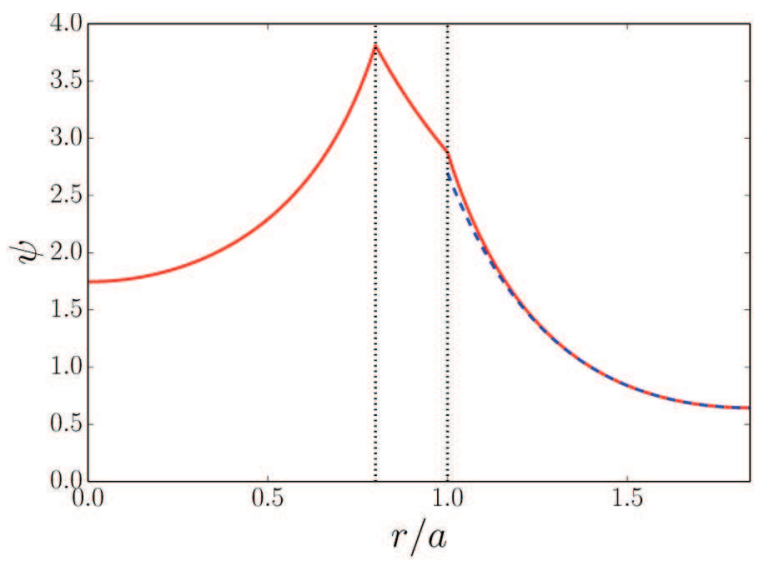

(a) Electrostatic potential profile computed inside and outside a hollow shell with the PB cell model (continuous red line) and outside a sphere with the DH cell model (dashed blue line), using the same outer radius, and a renormalized charge and an effective Debye length such that the two solutions match on the cell boundary at $r=R$.

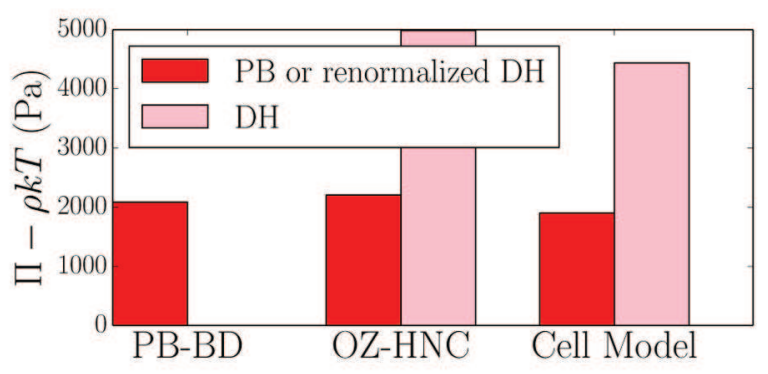

(b) Osmotic pressures predicted with different models for highly charged hollow shells. PB-BD: Brownian dynamics coupled to the resolution of the non-linear $\mathrm{PB}$ equation; OZ-HNC dark red: with renormalization; OZHNC light pink: with un-renormalized potential (10)(11); Cell Model dark red: based on PB equation; Cell Model light pink: based on the DH equation without renormalization.

Figure 8. Illustration of the renormalization procedure (top) and results (bottom) for $a=14 \mathrm{~nm}, \alpha=0.8, \phi=0.16, \epsilon_{\mathrm{s}}=2, \kappa a=2, \bar{\sigma}=5$, leading to $\kappa_{\text {eff }}=2.204 a$ and $Z_{\text {eff }}=152.16\left(\bar{\sigma}_{\text {eff }}=3.61\right)$.

volume fraction and the surface charge are quite high. The OZHNC theory based on a Yukawa pair potential with parameters obtained from the renormalization procedure presented above is, however, even more precise. The small difference with respect to the $\mathrm{PB}-\mathrm{BD}$ simulation is within the $\sim 5 \%$ uncertainty due to numerical convergence.

To conclude, we have shown that the pair potential (10)(11) based on the DH theory should not be used for highly charged hollow shells, just as the Yukawa potential with a bare charge should not be used for full spheres. Nevertheless, it is still possible to use DH-like theories based on a Yukawa potential with an effective charge and Debye length to model the structure and thermodynamics of suspensions of highly charged hollow shells. These effective parameters can be obtained in a simple manner with the cell model renormalization procedure presented above. As for full sphere systems, this approach is valid if the typical interparticle distance is larger than the Debye length.

\section{CAPSULE LOADING WITH IONIC SPECIES}

The aim of the previous section was to provide ways to efficiently model colloid-colloid interactions and their effect on macroscopic quantities such as an equation of state. These interactions can be tuned by acting on the shell thickness or permittivity to build materials with the desired properties. Hollow shells can also be used as nanoreservoirs or nanovectors containing small charged species. In this section, we focus on the loading and unloading of small charged species in porous, charged, hollow shells.

This transfer can be achieved following two routes. The (electro)chemical potential of ions is $k T \ln \left(n^{ \pm}\right) \pm e \Psi$ in the PB theory for 1:1 electrolytes, and equal to $k T \ln n_{0}$ at equilibrium. Modifying the ion density inside the capsules, $n_{\mathrm{in}}^{ \pm}$, is therefore possible either by changing the imposed chemical potential, $k T$ $\ln n_{0}$, or by altering the electrostatic potential field, $\Psi_{\text {in }}$ inside the capsule. If the system is in contact with an ion reservoir, the first solution consists in changing the salt concentration of the latter. In the other case, it consists in modifying the bulk ion concentration for example by adding salt or solvent to a closed system. Loading or unloading charged species by acting on the field $\Psi_{\text {in }}$ can be achieved either by changing the surface charge of the hollow shells (with a $\mathrm{pH}$ change for example) or by modifying their volume fraction, if we rule out modifications of their thickness or permittivity, which may prove difficult and/or irreversible.

Counterion density distributions were computed for spheres and for hollow shells with $\alpha=0.8$ both with the cell model and with PB-BD simulations for $\phi=0.02$ and $0.2, \kappa a=0.5$ and 2, and $\epsilon_{s}=2$ and 78. In the PB-BD simulations, the distributions were obtained by averaging the local ion density profiles computed around each of the $N$ colloids in the simulation box. One example of ion distribution is reported in Figure 9. The difference in ion density predicted by the cell model and the PB-BD simulations did not exceed $5 \%$ for the wide range of parameters investigated. This is a very interesting result since it proves the good quality of the predictions of the counterion density inside capsules by the cell model despite its erroneous evaluation of the osmotic pressure in liquid-like suspensions

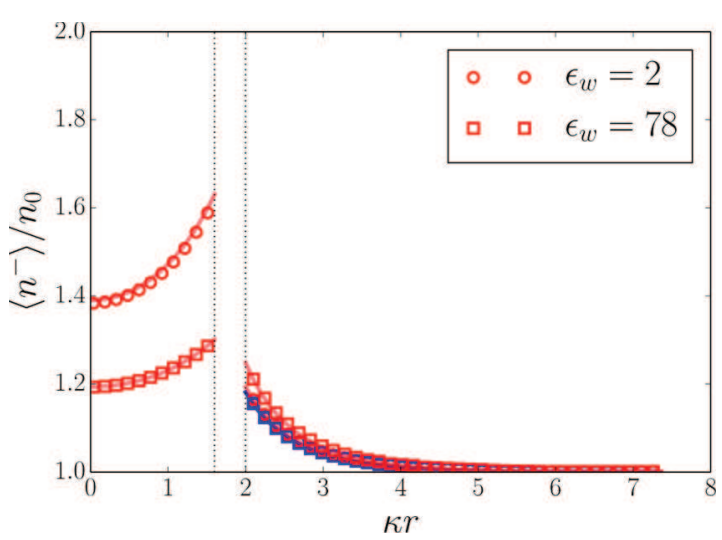

Figure 9. Average counterion concentration $\left\langle n^{-}\right\rangle$as a function of the distance from the center of the colloids. Light red: shells; dark blue: spheres; symbols: PB-BD simulations; lines: cell model. The dotted lines materialize the positions of the inner and outer surfaces of the shells. $a=14 \mathrm{~nm}, \phi=0.02, \kappa a=2, \alpha=0.8, \epsilon_{\mathrm{s}}=2 \epsilon_{0}$, and $\bar{\sigma}=\bar{\sigma}^{\prime}=0.25$. 
discussed previously. The cell model will therefore be used to discuss the electrostatic loading and unloading of hollow shells in what follows.

In the three following sections, the focus will be on the dependence of average co- and counterion concentrations inside and outside capsules on the reservoir ion concentration, the volume fraction, and the surface charge density. In the cell model, these quantities are defined by

$$
\begin{aligned}
& \left\langle I_{\text {in }}^{ \pm}\right\rangle=\frac{3 I_{0}}{a^{\prime 3}} \int_{0}^{a^{\prime}} r^{2} \mathrm{e}^{\mp \psi} \mathrm{d} r \\
& \left\langle I_{\text {out }}^{ \pm}\right\rangle=\frac{3 I_{0}}{R^{3}-a^{3}} \int_{a}^{R} r^{2} \mathrm{e}^{\mp \psi} \mathrm{d} r
\end{aligned}
$$

4.1. Influence of the Reservoir Salt Concentration. The typical evolution of $\left\langle I^{ \pm}\right\rangle$as a function of the salt reservoir concentration, $I_{0}$, is represented in Figure 10a.

In the high-salt regime, electrostatics are strongly screened and the ion densities both inside and outside capsules tend toward the ion reservoir density, $\left\langle I^{ \pm}\right\rangle=I_{0}$.

For slightly lower salt concentrations, electrostatics influence ionic density profiles but the $\mathrm{PB}$ equation can be linearized. In this case, the potential follows relation (7) and the integrals in (15)-(16) can be computed numerically. The result is represented with thin lines in Figure 10a. As expected, the agreement with the nonlinear PB solution is good only in the quite strongly screened regime.

In the low-salt regime, the electrostatic potential field and the counterion density asymptote to the solution of the nonlinear $\mathrm{PB}$ equation for the no-salt regime $\psi_{\mathrm{ns}}$ and the counterion density $n_{\mathrm{ns}}$. Since the counterion density tends to a value independent of $n_{0}$, and since relation $n^{+} n^{-}=n_{0}^{2}$ is always valid, $n^{+}$scales as $n_{0}{ }^{2}$ as verified in Figure 10a. We must emphasize the necessity of using the nonlinear PB equation to explore the low-salt regime. Since the interior of a capsule is a cavity with charges facing each-other, and since the EDL thickness tends to infinity as $n_{0} \rightarrow 0$, the linear $\mathrm{PB}$ equation predicts an electrostatic potential and a counterion concentration diverging to infinity in the low-salt regime, which is clearly unphysical (see thin lines in Figure 10a). Note that it is necessary to consider the full nonlinear $\mathrm{PB}$ equation even if the dimensionless charge $Z l_{\mathrm{B}} / a$ is as low as 0.5 here. It is sometimes mentioned in the literature that, as a rule of thumb, the $\mathrm{PB}$ equation can be linearized for $Z l_{\mathrm{B}} / a \lesssim 10$. This is obviously not the case here. The reason is that, in the low salt regime, the EDL are much larger than colloids $(\kappa a \ll 1)$, so the pertinent length scale to nondimensionalize the $\mathrm{PB}$ equation is the Debye length and not the colloid size. In this case, the boundary condition on the surface of colloids relates the normal component of the electric field to $\bar{\sigma}$ and not to $Z l_{\mathrm{B}} / \mathrm{a}$, with $\bar{\sigma}$ scaling as $n_{0}{ }^{-1 / 2}$, and thus diverging in the low-salt regime however low the real surface charge is.

From a practical point of view, the results displayed in Figure 10a emphasize two points: (i) The use of the $\mathrm{DH}$ equation leads to the unphysical conclusion that a very large amount of counterions could be stored in charged capsules thanks to electrostatic effects if the electrolyte is partially deionized. In contrast, the nonlinear PB theory predicts a constant asymptote for the counterion concentration, so the capsules cannot be significantly loaded at low salt concentrations, and can never be fully emptied either. The minimum amount of counterions trapped inside the hollow shells is given by the no-salt solution of the nonlinear PB equation, which is not analytical. (ii) The

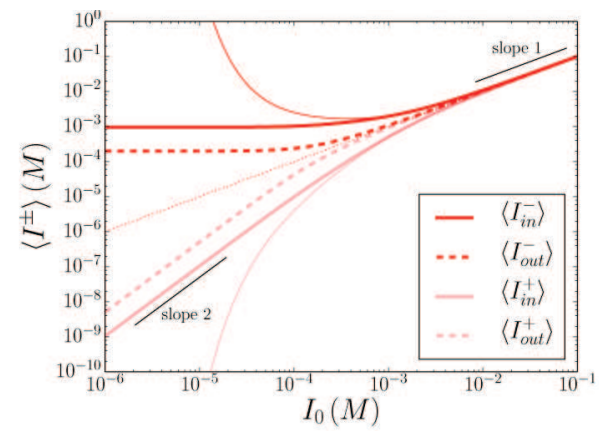

(a) Effect of the salt reservoir concentration $I_{0} . \phi=0.1, Z l_{B} / a=0.5$.

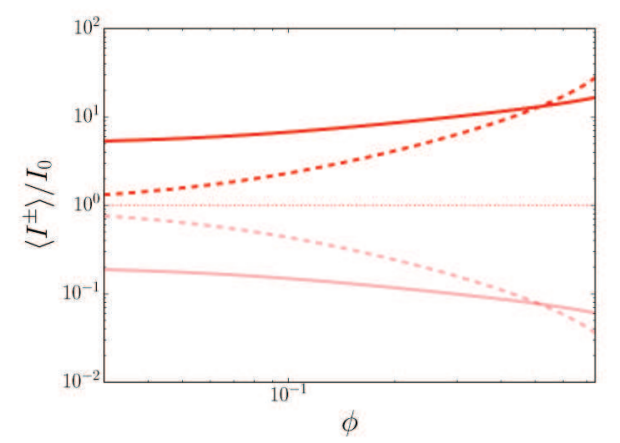

(b) Effect of the volume fraction $\phi . \kappa a=$ $0.5, \bar{\sigma}=1$.

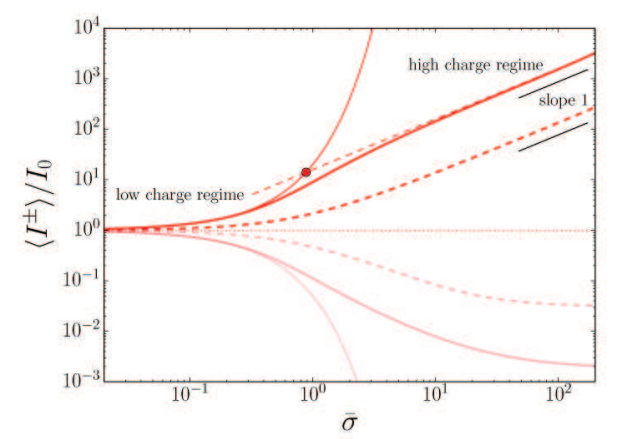

(c) Effect of the dimensionless surface charge density $\bar{\sigma}$. Thin dashed line: relation (17). The symbol is located at $\bar{\sigma}=\bar{\sigma}_{t}$, which corresponds to the transition between the low and high charge regimes. $\phi=0.1, \kappa a=0.5$

Figure 10. Average counterion (rep. co-ion) concentration $\left\langle I^{-}\right\rangle$ (respectively $\left\langle I^{+}\right\rangle$) inside and outside capsules (subscripts in and out respectively) as a function of the salt reservoir concentration $I_{0}$, volume fraction $\phi$, and dimensionless charge density $\bar{\sigma}$. Thick lines: PB cell model. Thin lines: DH cell model (7). Dotted line: uncharged limiting case $\left\langle I^{+}\right\rangle=\left\langle I^{-}\right\rangle=I_{0}$. Parameters common to every figure: $a=$ $14 \mathrm{~nm}, \alpha=0.8$, and $\epsilon_{s}=2 \epsilon_{0}$.

average salt concentration that can actually be loaded into a capsule with electrostatic effects is a monotonic increasing function of the salt reservoir concentration, with a slope varying between zero close to the no-salt regime and one in the highsalt limit.

4.2. Influence of the Volume Fraction. The effect of the volume fraction on the average ion concentrations inside and outside a capsule can be observed in Figure 10b. The co- and 
counterion average concentrations inside capsules hardly depend on the volume fraction. The striking effect of the volume fraction is on the counterion concentration outside capsules, which increases by 2 orders of magnitude between $\phi$ $=0.01$ and 0.9 and becomes greater than that inside capsules for $\phi \simeq 0.55$ here. The co-ion concentration outside capsules also decreases faster than that inside capsules, so, at high volume fraction, the co-ions are more concentrated inside capsules. Hence, charged hollow shells can be counterion reservoirs at low volume fractions or co-ion reservoirs near close packing. This effect was discovered as early as 1977 by Mille and Vanderkooi, ${ }^{5}$ but it is mentioned here for completeness. The reason for this reversal is the increase of the electrostatic potential outside the capsules when the dispersion is progressively concentrated, this increase being linked to the diminishing average distance between the outer surfaces of the capsules. The reversal of the reservoir behavior is thus expected at volume fractions such that the inter particle distance is of the order of the inner shell radius. However, there is no point in trying to determine a more quantitative picture with the cell model. At such high volume fractions, the correlations of positions between shells become important since they determine the exact geometry of the charged surfaces acting as boundary conditions for the electrostatic potential field. It would be necessary to compute average ion concentrations for various structures, such as BCC, FCC, etc. This is a vast task that we leave for future studies. In what follows, the volume fraction is low enough for the capsules to remain counterion reservoirs.

4.3. Influence of Capsule Surface Charge. We now turn to the dependence of ion loading on the surface charge, as represented in Figure 10c.

At vanishingly small surface charge, the ion concentration is uniform and equal to $I_{0}$ as expected. For small but nonzero surface charges, the counterion and co-ion average concentrations inside capsules are very well represented by a numerical evaluation of $(15)-(16)$ with the analytical solution of the $\mathrm{DH}$ eq 7 , here valid for $\bar{\sigma}<\bar{\sigma}_{t} \simeq 1$ (thin lines).

Interestingly, the inner and outer counterion concentrations exhibit a linear dependence on the colloidal charge in the high charge regime $\left(\bar{\sigma}>\bar{\sigma}_{t}\right)$. This is a signature of the onset of ionic condensation on the surface of the capsules. When the surface charge becomes very large, the electrostatic potential field tends to the field $\psi^{\text {sat }}$ in a region $\Omega$ far away from the surface, $\psi^{\text {sat }}$ being independent of the surface charge. The number of ions inside this region is then $N_{\text {sat }}^{ \pm}=\int_{\Omega} n_{0} e^{\mp \psi \text { sat }} \mathrm{d} V$ and is thus also independent of $Z$. Close to the interface, the potential continues to evolve and a new counterion is "condensed" in this region as soon as a new charge is added to the surface. ${ }^{36}$ The number of ions in this layer is therefore $N_{\text {cond }}^{-}=\gamma Z$ in the high charge regime, where $\gamma$ is a constant. The number of ions inside capsules is thus $N^{\text {sat }}+\gamma Z$ in the condensation regime. For large surface charge values the second term is large compared to the first one, which explains the observations made from Figure 10c: $\left\langle I_{\text {in }}^{-}\right\rangle \propto Z$ and $\left\langle I_{\text {out }}^{-}\right\rangle \propto Z$ in the high charge limit. We also observe that higher surface charges are required for this behavior to set in for the counterion concentration outside capsules. This is not surprising since the electrostatic potential field is lower outside capsules than inside them.

There is, of course, no co-ion condensation. Co-ions are actually quite strongly expelled from the region in which counterions are condensed, so the number of co-ions tends to the constant value $N_{\text {sat }}^{+}$for very high surface charges. This is the reason for the horizontal asymptotes of $\left\langle I_{\text {in }}^{+}\right\rangle$and $\left\langle I_{\text {out }}^{+}\right\rangle$in Figure $10 \mathrm{c}$ for $\sigma^{-} \gtrsim 10-100$. Note that the fact that the total number of counterions in the condensed layer scales as $Z$ does not imply that the counterion density profile inside this layer scales as $Z$, so the relation $n^{+} n^{-}=n_{0}^{2}$ obeyed locally cannot be used to infer a relation like $N_{\text {cond }}^{+} \propto 1 / Z$. If it is assumed that the entire $n^{-}$profile scales as $Z$, then the average co-ion concentration $\left\langle I^{+}\right\rangle$would be $N_{\text {sat }}^{+} / V_{\text {in }}+\delta /(\gamma Z)$, where $\delta$ is a constant independent of $Z$. Although there is no rigorous proof for this functional form, the data represented in Figure 10c supports it for the range of surface charge in which $\left\langle I^{-}\right\rangle$is linear.

Estimating the counterion average concentration inside a capsule in the high surface charge regime is extremely important since it is the regime to be used to store a maximum amount of counterions. We could not derive the corresponding analytical expression because it requires the solution of the nonlinear PB equation. However, the parameter space ( $a, \phi, \alpha$, $\left.I_{0}, \epsilon_{s}\right)$ was explored numerically to determine an empirical correlation for $\left\langle I_{\text {in }}^{-}\right\rangle(\bar{\sigma})$ in the high-charge regime. We found that the slope does not depend on $\phi$ and $\epsilon_{s}$, and scales as $I_{0}^{-1}$, $a^{-2}$, and $\alpha^{-1}$. These observations lead to the following expression for the counterion average concentration inside highly charged capsules

$$
\left\langle I_{\text {in }}^{-}\right\rangle=\frac{c}{\kappa a \kappa a^{\prime}} \bar{\sigma}=\frac{c}{\alpha(\kappa a)^{2}} \bar{\sigma} \text { for } \bar{\sigma}>\bar{\sigma}_{t}
$$

where $c \simeq 6.1 \pm 0.05$ is a constant determined numerically and $\bar{\sigma}_{t} \sim 1$ is the threshold dimensionless charge above which this regime is observed.

To conclude this part, we have shown that the average concentration of counterions that can be stored inside the internal EDL of a capsule increases monotonically with the surface charge density, as could be expected. Relations have been provided to compute this quantity both in the low charge regime, thanks to eqs 7 and 15, and in the high charge regime corresponding to $\bar{\sigma} \gtrsim 1$, thanks to eq 17 . The later regime is most interesting to entrap a large quantity of ionic species inside capsules.

\section{CONCLUSION}

Suspensions of colloidal, porous, and charged hollow shells are involved in many biological systems and in several new and interesting applications such as nanovectorization, sensing, or photonics. Understanding the characteristics and behavior of these suspensions is necessary at two levels. At the meso- or macro-scale, the structure, stability, thermodynamics, and rheology of suspensions is largely determined by (possibly many-body) colloid-colloid interactions. At the microscopic scale, the ion-colloid interactions determine the capacity of hollow shells to store ions in the shells' often enhanced electric double layers. There is therefore a strong need to understand the electrostatics of charged hollow shell suspensions. In the present article, we have considered potentially concentrated suspensions of hollow shells with a uniform surface charge density but finite and arbitrary thickness and permittivity.

The Debye-Hückel (DH) limit was explored. The analytical solution in the cell model was derived, showing that very high electrostatic potentials can be obtained inside hollow colloids when their permittivity is low and when the inner (core) radius is decreased. These high potentials are desirable, for example, in order to trap a significant amount of counterions inside hollow shells. For an infinite cell radius, the analytical solution already 
derived for an isolated shell is recovered. ${ }^{14}$ We used this solution with the linear superposition approximation to propose an analytical model for pair interactions between hollow shells valid in dilute suspensions of weakly charged colloids. This model is actually a simple Yukawa potential based on the usual Debye length but with an effective surface charge given by relation (10). This is a very useful result since classical theories and simulations based on pair potentials and devised for full spheres can be employed straightforwardly for hollow shells by replacing the surface charge by the effective charge (10). This pair interaction model has been validated against numerical simulations of the 3D fully coupled Debye-HückelLaplace electrostatics problem. Image effects were shown to exist, but to be of the same order as the ones usually neglected for full spheres.

The next step, beyond the two-body level, is to be able to predict the macroscopic behavior of a suspension. Equations of state obtained with the Ornstein-Zernike equation with the Rogers-Young (RY) closure (OZ-RY) were compared to reference predictions of Brownian dynamics simulations coupled to a full resolution of the N-body Debye-HückelLaplace problem at each time step. As expected, the classical integral equation theory performs very well for dilute suspensions with the pair potential derived for isolated pairs of colloids in the first section of this work. In concentrated suspensions, many-body effects were measured for both spheres and hollow shells, showing that full spheres require a manybody correction to the pair potential due to volume exclusion of the ions from a third particle's core, ${ }^{35,36}$ while, interestingly, this correction is not always necessary for hollow shells. The correction is required only for hollow shells with a significant thickness and/or a low shell permittivity. In many applications, shells are thin enough for many-body effects to be neglected even in quite concentrated suspensions, and the integral equation theory based on isolated pair potentials like (10) is actually even more efficient than for spheres. For significantly charged colloids, the $\mathrm{DH}$ picture fails, so a charge renormalization method based on the cell model was proposed and validated against nonlinear Poisson-Boltzmann-Brownian dynamics simulations.

Finally, we have discussed the problem of predicting the quantity of ions that can be electrostatically "trapped" into hollow shells for nanocontainer applications. This topic has already been considered within the cell model, but without justification of the validity of this approach. In this work, manybody simulations first showed that the cell model provides quite good estimates of the ion concentration profiles inside and outside hollow shells both in concentrated and dilute regimes (whereas it is known to predict wrong pressures in dilute suspensions). The dependence of the average counterion concentration inside shells $\left\langle I_{\text {in }}^{-}\right\rangle$was discussed as a function of three parameters that can be used to trigger counterion loading or unloading, namely the salt concentration $I_{0}$ (where the potential vanishes), the volume fraction (modified during film drying, filtration or dialysis for instance), and the surface charge of the shells (modified with $\mathrm{pH}$ changes for instance). $\left\langle I_{\text {in }}^{-}\right\rangle$is a monotonic, increasing function of $I_{0}$ with a plateau at low salt concentrations corresponding to the no-salt solution (i.e., particles cannot be completely unloaded) and a slope 1 at large salt concentration indicating a complete screening of electrostatics and a loading mechanism due to pure diffusion. It has been shown that $\left\langle I_{\text {in }}^{-}\right\rangle$increases with volume fraction, which is simply a Donnan effect, but that the average counterion concentration outside shells increases faster due to the very small intercolloid distances near close packing. This effect, already mentioned in the literature, ${ }^{5}$ can be used to store coions inside charged capsules upon concentration of the suspension for example during the drying of a nanocontainer based coating. Finally, it has been shown that variations of $\left\langle I_{\text {in }}^{-}\right\rangle$ with the surface charge density of hollow shells can be completely predicted with the analytical solution of the cell model in the DH limit and by relation (17) in the high charge regime, the transition being at $\sigma \approx \sqrt{2 n_{0} \epsilon k T}$.

A few modeling choices have been made in this work in order to keep the results as generic and tractable as possible, and some refinements are of course still possible. The main issues for general applications are the use of the PoissonBoltzmann theory and of the constant charge boundary conditions. As mentioned before, the domain of validity of the PB theory is now well established and apart from a few exceptions, it corresponds to $1: 1$ electrolytes. For multivalent (counter-)ions, this theory may yield neither quantitative nor qualitative results because of ion-ion correlations. It would therefore be quite useful to extend the results presented in this work to multivalent electrolytes, using integral equation and molecular dynamics or Monte Carlo simulations based on the primitive model. The effect of the finite size of the ions, important at high salt concentrations, may be explored with these methods or more simply with the modified PB equation. The constant charge boundary condition was used for simplicity, for comparison of hollow shell suspensions with sphere suspensions for which many results are available for constant surface charges, and to keep the presentation quite generic. Since a number of surfaces actually exhibit charge regulation, and since it is a mechanism which could prove useful to trigger the loading or unloading of nanocontainers, it would indeed be interesting to determine if and how the results of sections 2 and 4.3 are modified by charge regulation mechanisms, for example with the recent modeling proposed by Trefalt and co-workers. ${ }^{26}$

Despite these limitations, we are convinced the simple and fast modeling strategies developed and validated in this work will help to improve the design and optimization of hollow shell suspensions for many new and exciting applications.

\section{PRESSURE COMPUTATION IN SIMULATIONS RESOLVING THE FULL ELECTROSTATIC POTENTIAL FIELD}

In Poisson-Boltzmann Brownian dynamics simulations, colloids are represented explicitly, but ions are only present implicitly. The pressure in the suspension can be obtained from the virial equation, including colloids and ions, as ${ }^{41}$

$$
P=\rho_{\mathrm{c}} \mathrm{kT}+P_{\mathrm{cc}}+P^{\prime}
$$

where

$$
P^{\prime}=\rho_{\text {ion }} \mathrm{kT}+P_{\text {ic }}+\frac{U}{3 V}
$$

$\rho_{\mathrm{c}} \mathrm{V}$ and $\rho_{\text {ion }} V$ are the number of colloids and ions in the total volume $V, P_{\mathrm{cc}}$ and $P_{\text {ic }}$ are the contributions of hard core collisions between colloids and between ions and colloids, respectively, and $U$ is the electrostatic energy. There is no ionion collision contribution in the PB theory. The colloid-colloid collision contribution is calculated in a classical pairwise manner 


$$
\bar{P}_{\mathrm{cc}}=\frac{P_{\mathrm{cc}}}{2 n_{0} k T}=-\frac{1}{3 \bar{V}} \sum_{i<j \in c} \overline{\mathbf{r}}_{i j} \cdot \overline{\mathbf{F}}_{i j}^{c}
$$

An overbar denotes a dimensionless quantity. The Debye length is chosen as the length scale, the ion reservoir pressure $2 n_{0} k T$ is chosen as the pressure scale, and $2 n_{0} k T \kappa^{-2}$ is the force scale. The collision force $\bar{F}_{i j}^{c}$ is computed with the algorithm presented by Foss and Brady ${ }^{42}$ for Brownian hard spheres. The forms of the other contributions to the pressure in (19) are given below in the framework of the PB theory for a 1:1 electrolyte. The local ion density in the suspension is $n^{+}+n^{-}=$ $2 n_{0} \cosh \psi$ and thus the ion density in the global volume $V$ is

$$
\bar{\rho}_{\text {ion }}=\frac{\rho_{\text {ion }}}{2 n_{0}}=\frac{1}{\bar{V}} \int_{\bar{V}_{\mathrm{e}}} \cosh \psi \mathrm{d} \bar{V}
$$

where $\bar{V}_{\mathrm{e}}$ is the volume occupied by the electrolyte. The ioncolloid collision contribution is given by a relation similar to (20) but with the ions represented by a continuous field. The force exerted by ions $j$ on a surface element $\mathrm{d} S$ of one colloid $c$ and due to hard core contacts is the ion pressure multiplied by $\mathrm{d} S$, i.e., $\mathbf{d f}_{c j}=-\left(n^{+}+n^{-}\right) k T \mathbf{n} \mathrm{d} S=-2 n_{0} k T \cosh \psi \mathbf{n} \mathrm{d} S$, where $\mathbf{n}$ is the unit vector normal to the surface and pointing toward the electrolyte. Therefore, relation 20 applied to ion-colloid collisions becomes

$$
\bar{P}_{\mathrm{ic}}=\frac{P_{\mathrm{ic}}}{2 n_{0} k T}=\frac{1}{3 \bar{V}} \sum_{c} \int_{\bar{S}_{c}} \cosh \psi \overline{\mathbf{x}}_{c} \cdot \mathbf{n} \mathrm{d} \bar{S}
$$

where $\boldsymbol{x}_{c}$ is the vector from the center of colloid $c$ to the surface element $\mathrm{d} S$. The electrostatic energy is computed as the contribution from every charge immersed in the potential field. The contribution of the fixed surface charges of the colloids is the integral of $\sigma \Psi$ on all colloidal surfaces. The contribution from the charges of the ions in the small volume $\mathrm{d} V$ is $\left(n^{+}-\right.$ $\left.n^{-}\right) e \Psi \mathrm{d} V=-2 n_{0} k T \psi$ sinh $\psi \mathrm{d} V$, so, after integration

$$
\bar{U}=\frac{U}{2 n_{0} k T \kappa^{-3}}=\sum_{c} \int_{\bar{S}_{c}} \frac{1}{2} \bar{\sigma} \psi \mathrm{d} \bar{S}-\int_{\bar{V}_{e}} \frac{1}{2} \psi \sinh \psi \mathrm{d} \bar{V}
$$

The pressure computed with (18) is not the osmotic pressure. In particular, it contains the ion reservoir pressure and the self-energy of the colloids. We therefore define the osmotic pressure as the excess pressure compared to the reservoir pressure, minus the self-contributions

$$
\begin{aligned}
\bar{\Pi}=\frac{\Pi}{2 n_{0} k T}=\overline{\rho_{c}} & +\frac{1}{\bar{V}} \int_{\bar{V}_{e}}(\cosh \psi-1) \mathrm{d} \bar{V} \\
& +\frac{1}{3 \bar{V}} \sum_{c} \int_{\bar{S}_{c}}(\cosh \psi-1) \overline{\mathbf{x}}_{c} \cdot \mathbf{n} \mathrm{d} \bar{S} \\
& -\frac{1}{3 \bar{V}} \int_{\bar{V}_{e}} \frac{1}{2} \psi \sinh \psi \mathrm{d} \bar{V} \\
& \mathrm{~d} \bar{S} \frac{1}{3 \bar{V}} \sum_{c} \int_{\bar{S}_{c}} \frac{1}{2} \bar{\sigma} \Psi \\
& -\frac{1}{3 \bar{V}} \sum_{i<j \in c} \bar{r}_{i j} \cdot \bar{F}_{i j}^{c} \\
& -\bar{P}^{\prime}{ }_{\text {self }}
\end{aligned}
$$

The $-2 n_{0} k T$ contribution necessary to switch from the pressure to the osmotic pressure is obtained with the " -1 " term in the first two integrals independently of the geometry of the colloids. It can be proven with the divergence theorem. We use this trick to improve the calculation accuracy as the terms $\bar{\rho}_{\text {ion }}+$ $\bar{P}_{\text {ic }}$ computed with (21) and (22) and the term $-2 n_{0} k T$ are often very large and almost cancel each other out, while we are only interested in the difference. Since the numerical integration generates errors of the order of $2-5 \%$, the present method yields a term $\bar{\rho}_{\text {ion }}+\bar{P}_{\text {ic }}-2 n_{0} k T$ directly, with a $2-5 \%$ error whereas this error could be as large as $100 \%$ using (21) and (22) and removing the reservoir pressure analytically. The term $P_{\text {self }}^{\prime}$ is $E / 3 V$, where $E$ is the energy of the system of $N_{c}$ colloids at infinite dilution. We evaluate $E$ as $N_{c} 3 V_{\phi \rightarrow 0} P_{\phi \rightarrow 0}^{\prime}$ independently of $\mathrm{BD}$ simulations, thanks to a $\mathrm{PB}$ computation with one fixed colloid in a large enough volume $V_{\phi \rightarrow 0}$ and with $P_{\phi \rightarrow 0}^{\prime}$ computed as the sum of the four terms containing integrals in (24).

It is worth mentioning that the expression (24) is a completely general result, only restricted to the hypotheses associated with the PB theory for a 1:1 electrolyte. In particular, it is valid for any colloid geometry and for heterogeneous surface charge densities. In the $\mathrm{DH}$ theory, the osmotic pressure is given by (24) with $\cosh \psi$ replaced by $1-\psi^{2} / 2$ and $\sinh \psi$ replaced by $\psi$. A similar form was used by Fushiki ${ }^{43}$ in the no-salt regime.

\section{AUTHOR INFORMATION}

\section{Corresponding Author}

*E-mail: yannick.hallez@univ-tlse3.fr.

\section{Notes}

The authors declare no competing financial interest.

\section{ACKNOWLEDGMENTS}

This work was performed using HPC resources from CALMIP (Project p1114) and GENCI (IDRIS/CINES, Grant x2016097003).

\section{REFERENCES}

(1) Caruso, F. Hollow Capsule Processing through Colloidal Templating and Self- Assembly. Chem. - Eur. J. 2000, 6, 413-419.

(2) Liu, J.; Liu, F.; Gao, K.; Wu, J.; Xue, D. Recent developments in the chemical synthesis of inorganic porous capsules. J. Mater. Chem. 2009, 19, 6073-6084.

(3) Antipov, A. A.; Sukhorukov, G. B. Polyelectrolyte multilayer capsules as vehicles with tunable permeability. Adv. Colloid Interface Sci. 2004, 111, 49-61. Plenary and Invited Lectures From the \{XVIth European Chemistry at Interfaces Conference, Vladimir, Russia, May 2003.

(4) Graf, C.; van Blaaderen, A. Metallodielectric Colloidal Core-Shell Particles for Photonic Applications. Langmuir 2002, 18, 524-534.

(5) Mille, M.; Vanderkooi, G. Electrochemical properties of spherical polyelectrolytes: II. Hollow sphere model for membranous vesicles. J. Colloid Interface Sci. 1977, 61, 455-474.

(6) Zhang, L.; D’Acunzi, M.; Kappl, M.; Auernhammer, G. K.; Vollmer, D.; van Kats, C. M.; van Blaaderen, A. Hollow Silica Spheres: Synthesis and Mechanical Properties. Langmuir 2009, 25, 2711-2717. PMID: 19437752.

(7) Zhang, L.; D’Acunzi, M.; Kappl, M.; Imhof, A.; Blaaderen, A. v.; Butt, H.-J.; Graf, R.; Vollmer, D. Tuning the mechanical properties of silica microcapsules. Phys. Chem. Chem. Phys. 2010, 12, 15392-15398.

(8) Dinsmore, A.; Hsu, M. F.; Nikolaides, M.; Marquez, M.; Bausch, A.; Weitz, D. Colloidosomes: selectively permeable capsules composed of colloidal particles. Science 2002, 298, 1006-1009. 
(9) Cordova, A.; Deserno, M.; Gelbart, W. M.; Ben-Shaul, A. Osmotic Shock and the Strength of Viral Capsids. Biophys. J. 2003, 85, $70-74$.

(10) Lee, S.-Y.; Lim, J.-S.; Harris, M. T. Synthesis and application of virus-based hybrid nanomaterials. Biotechnol. Bioeng. 2012, 109, 1630.

(11) Rong, J.; Niu, Z.; Lee, L. A.; Wang, Q. Self-assembly of viral particles. Curr. Opin. Colloid Interface Sci. 2011, 16, 441-450.

(12) Angelescu, D. G.; Caragheorgheopol, D. Influence of the shell thickness and charge distribution on the effective interaction between two like-charged hollow spheres. J. Chem. Phys. 2015, 143, $14490210.1063 / 1.4932372$

(13) Marcus, R. A. Calculation of Thermodynamic Properties of Polyelectrolytes. J. Chem. Phys. 1955, 23, 1057-1068.

(14) Šber, A.; Podgornik, R. Role of electrostatic interactions in the assembly of empty spherical viral capsids. Phys. Rev. E 2007, 76, 061906

(15) Shojaei, H. R.; LoŠdorfer Božič, A.; Muthukumar, M.; Podgornik, R. Effects of long-range interactions on curvature energies of viral shells. Phys. Rev. E: Stat. Phys., Plasmas, Fluids, Relat. Interdiscip. Top. 2016, 93, 052415.

(16) Feitosa, E.; Neto, A. A.; Chaimovich, H. Integration of the nonlinear Poisson-Boltzmann equation for charged vesicles in electrolytic solutions. Langmuir 1993, 9, 702-707.

(17) Stukan, M. R.; Lobaskin, V.; Holm, C.; Vinogradova, O. I. Spatial distribution of polyelectrolyte and counterions in nanocapsules: A computer simulation study. Phys. Rev. E 2006, 73, 021801.

(18) Oberdisse, J.; Porte, G. Size of microvesicles from charged surfactant bilayers: Neutron scattering data compared to an electrostatic model. Phys. Rev. E: Stat. Phys., Plasmas, Fluids, Relat. Interdiscip. Top. 1997, 56, 1965-1975.

(19) Oberdisse, J. Transition from small to big charged unilamellar vesicles. Eur. Phys. J. B 1998, 3, 463-469.

(20) Lošdorfer Božič, A.; Podgornik, R. Symmetry effects in electrostatic interactions between two arbitrarily charged spherical shells in the Debye-Hückel approximation. J. Chem. Phys. 2013, 138, 074902

(21) Israelachvili, J. N. Theoretical considerations on the asymmetric distribution of charged phospholipid molecules on the inner and outer layers of curved bilayer membranes. Biochim. Biophys. Acta, Biomembr. 1973, 323, 659-663.

(22) Hedrick, M. M.; Chung, J. K.; Denton, A. R. Structure and osmotic pressure of ionic microgel dispersions. J. Chem. Phys. 2015, $142,034904$.

(23) Tang, Q.; Denton, A. R. Ion density deviations in semipermeable ionic microcapsules. Phys. Chem. Chem. Phys. 2015, 17, 11070-11076.

(24) Yang, J.; Lee, J.; Kang, J.; Lee, K.; Suh, J.-S.; Yoon, H.-G.; Huh, Y.-M.; Haam, S. Hollow silica nanocontainers as drug delivery vehicles. Langmuir 2008, 24, 3417-3421.

(25) Naji, A.; Jungblut, S.; Moreira, A. G.; Netz, R. R. Electrostatic interactions in strongly coupled soft matter. Phys. A (Amsterdam, Neth.) 2005, 352, 131-170. Physics Applied to Biological SystemsPhysics Applied to Biological Systems.

(26) Trefalt, G.; Behrens, S. H.; Borkovec, M. Charge regulation in the electrical double layer: ion adsorption and surface interactions. Langmuir 2016, 32, 380-400. PMID: 26599980.

(27) Hallez, Y.; Diatta, J.; Meireles, M. Quantitative Assessment of the Accuracy of the Poisson-Boltzmann Cell Model for Salty Suspensions. Langmuir 2014, 30, 6721-6729. PMID: 24834492.

(28) Russel, W. B.; Saville, D. A.; Schowalter, W. R. Colloidal dispersions; Cambridge University Press: Cambridge, U.K., 1989.

(29) Bell, G.; Levine, S.; McCartney, L. Approximate methods of determining the double-layer free energy of interaction between two charged colloidal spheres. J. Colloid Interface Sci. 1970, 33, 335-359.

(30) Ohshima, H. Electrostatic Interaction between Two Dissimilar Spheres: Image-Interaction Correction to the Linear Superposition Approximation. J. Colloid Interface Sci. 1995, 176, 7-16.
(31) Deserno, M.; von Grünberg, H.-H. Osmotic pressure of charged colloidal suspensions: A unified approach to linearized PoissonBoltzmann theory. Phys. Rev. E: Stat. Phys., Plasmas, Fluids, Relat. Interdiscip. Top. 2002, 66, 011401.

(32) Dobnikar, J.; Castaneda-Priego, R.; von Grünberg, H.; Trizac, E. Testing the relevance of effective interaction potentials between highly-charged colloids in suspension. New J. Phys. 2006, 8, 277.

(33) Trizac, E.; Belloni, L.; Dobnikar, J.; von Grünberg, H. H. Castañeda-Priego, R. Macroion virial contribution to the osmotic pressure in charge-stabilized colloidal suspensions. Phys. Rev. E 2007, 75, 011401 .

(34) Denton, A. R. Poisson-Boltzmann theory of charged colloids: limits of the cell model for salty suspensions. J. Phys.: Condens. Matter 2010, 22, 364108.

(35) Khan, S.; Morton, T. L.; Ronis, D. Static correlations in macroionic suspensions: Analytic and numerical results in a hypernettedchain mean-spherical approximation. Phys. Rev. A: At., Mol., Opt. Phys. 1987, 35, 4295-4305.

(36) Belloni, L. Colloidal interactions. J. Phys.: Condens. Matter 2000, 12, R549.

(37) Dobnikar, J.; Brunner, M.; von Grünberg, H.-H.; Bechinger, C. Three-body interactions in colloidal systems. Phys. Rev. E 2004, 69, 031402.

(38) Belloni, L. Ionic condensation and charge renormalization in colloidal suspensions. Colloids Surf., A 1998, 140, 227-243.

(39) Alexander, S.; Chaikin, P. M.; Grant, P.; Morales, G. J.; Pincus, P.; Hone, D. Charge renormalization, osmotic pressure, and bulk modulus of colloidal crystals: Theory. J. Chem. Phys. 1984, 80, 57765781 .

(40) Trizac, E.; Bocquet, L.; Aubouy, M.; von Grünberg, H. H. Alexander's Prescription for Colloidal Charge Renormalization. Langmuir 2003, 19, 4027-4033.

(41) Deserno, M.; Holm, C.; Kremer, K. Molecular dynamics simulations of the cylindrical cell model. Surf. Sci. Ser. 2001, 59-110.

(42) Foss, D. R.; Brady, J. F. Brownian Dynamics simulation of hardsphere colloidal dispersions. J. Rheol. 2000, 44, 629-651.

(43) Fushiki, M. Molecular-dynamics simulations for charged colloidal dispersions. J. Chem. Phys. 1992, 97, 6700-6713. 